\title{
Mathematical Analysis of a Single-Species Population Model in a Polluted Environment with Discrete Time Delays
}

\author{
Swarnali Sharma and G. P. Samanta \\ Department of Mathematics, Bengal Engineering and Science University, Shibpur, Howrah 711 103, India \\ Correspondence should be addressed to G. P. Samanta; g_p_samanta@yahoo.co.uk
}

Received 16 September 2012; Accepted 12 May 2013

Academic Editor: Liwei Zhang

Copyright (c) 2013 S. Sharma and G. P. Samanta. This is an open access article distributed under the Creative Commons Attribution License, which permits unrestricted use, distribution, and reproduction in any medium, provided the original work is properly cited.

\begin{abstract}
We have discussed the dynamical behaviour of a single-species population model in a polluted environment which describes the effect of toxicants on ecological system. Boundedness, positivity, and stability analysis of the model at various equilibrium points is discussed thoroughly. We have also studied the effect of single discrete delay as well as double discrete delays on the population model. Existence conditions of the Hopf bifurcation for single time delay are investigated. The length of delay preserving the stability is also estimated. The direction and the stability criteria of the bifurcating periodic solutions are determined by using the normal form theory and the center manifold theorem. The stability of the model with double time delays is investigated by using the Nyquist criteria. By choosing one of the delays as a bifurcation parameter, the model is found to undergo a Hopf bifurcation. Some numerical simulations for justifying the theoretical results are also illustrated by using MATLAB, which shows the reliability of our model from the practical point of view.
\end{abstract}

\section{Introduction}

In the world today, the pollution of the environment is a threatening problem due to the rapid development of industrialization. The presence of toxicants in the environment decreases the growth rate of species and its carrying capacity. In recent years, the most serious problem to society is the change in the environment caused by pollution, affecting the long-term survival of species, human life style, and biodiversity of the habitat. A large quantity of the toxicants and contaminants enter into the ecosystem one after another which seriously threaten the survival of the exposed population including humans. Therefore, the study of the effects of toxicants on the population and the assessment of the risk to populations are becoming quite important.

The problem of estimating qualitatively the effect of a toxicant on a population by mathematical models is a very effective way. The study of deterministic dynamic population models with toxicant effect was proposed by Hallam and his colleagues in 1980s [1-3]. This model was revisited by many researchers $[4,5]$. He and $\mathrm{Ma}$ in $[6,7]$ discussed the survival of a single-species in a polluted environment, considering the organism's uptake of toxicant from the environment and egestion of toxicant into the environment. In [4] Ma studied a Leslie resource-consumer model and obtained the threshold between the persistence and extinction of the consumer. In [8], Buonomo et al. studied the effect of variation of the population on the toxicant concentration in the organism and environment. Samanta and Maiti [9] studied a dynamical model of a single-species system in a polluted environment under two cases: constant exogenous input of toxicant and rapidly fluctuating random exogenous input of toxicant into the environment by means of ordinary and stochastic differential equations. There are many other works on the effects of a single toxicant on various ecosystems by using mathematical models [10-15].

Time delays have been incorporated in ecological models by many authors [16-27]. Introducing such delays in ecological models makes them more realistic and reasonable. Delay may have very complicated impact on the dynamics of a system since delay can cause the change of stability and can bifurcate periodic solutions.

In this paper, we have developed a single-species population model in polluted environment. In Section 2, we present a brief sketch of the construction of the model. Boundedness and positivity analysis of the solutions is shown 
which implies that the system is ecologically well behaved. In the next section, we have discussed the existence and stability analysis of various equilibrium points under zero-exogenous input and nonzero constant exogenous input. Next, we obtain necessary conditions for the existence of interior equilibrium $E^{*}$ and local and global stability of the system at $E^{*}$. The analysis of single delayed model is described in Section 4. This analysis shows that there is a critical value $\tau_{*}$ of delay $\tau$ below which the system is stable and above which the system becomes unstable at the interior equilibrium $E^{*}$. The system undergoes a Hopf bifurcation around $E^{*}$ at $\tau=\tau_{*}$. In Section 5, we have estimated the length of delay to preserve the stability around $E^{*}$. The direction and the stability criteria of the bifurcation periodic solutions by using the normal form theory and the center manifold theorem are discussed in Section 6. In the next section, the analysis of double delayed model is discussed. The stability of the model with double delays is investigated by the Nyquist criteria. By choosing one of the delays as a bifurcation parameter, the model is found to undergo a Hopf bifurcation. Some of the important analytic results are numerically verified by using MATLAB in Section 8. Finally, Section 9 contains the general discussions of the paper and ecological implications of our mathematical findings.

\section{Basic Mathematical Model}

In this paper, we analyze a model which describes the effect of toxicant on a single-species population. Here we assume the following:

$n(t)$ : concentration of the population biomass,

$c(t)$ : concentration of the toxicant in the population,

$s(t)$ : concentration of the toxicant in the environment.

The model satisfies the following assumptions.

(A1) There is a given toxicant in the environment and the living organisms absorb into their bodies part of this toxicant so that the dynamics of the population is affected by the toxicant.

(A2) For the growth rate of population, we assume that the birth rate is $b_{0}-f n(t)$ and the death rate is $d_{0}+\alpha c(t)$, where $b_{0}, f, d_{0}$, and $\alpha$ are assumed to be positive constants.

We consider the model

$$
\begin{gathered}
\frac{d n}{d t}=n\left(b_{0}-d_{0}-\alpha c-f n\right), \\
\frac{d c}{d t}=k s-\left(r+m+b_{0}-f n\right) c, \\
\frac{d s}{d t}=-k s n+\left(r+d_{0}+\alpha c\right) c n-h s+u(t),
\end{gathered}
$$

with initial data $n(0)>0, c(0)>0$, and $s(0)>0$. Here,

$k$ : depletion rate of toxicant in the environment due to its intake made by the population, $r$ : depletion rate of toxicant in the population due to egestion,

$m$ : depletion rate of toxicant in the population due to metabolization process,

$h$ : depletion rate of toxicant in the environment,

$u(t)$ : exogenous toxicant input rate which is assumed to be a smooth bounded nonnegative function of $t$.

We can see that if $b_{0}-d_{0}-\alpha c(t) \leq 0$, then $n(t)$ will be going to extinct. So we suppose that

$$
\begin{array}{r}
p=b_{0}-d_{0}>0, \quad b_{0}-d_{0}-\alpha c(t)>0 \\
\text { implies } c(t)<\frac{b_{0}-d_{0}}{\alpha}, \quad \forall t \geq 0 .
\end{array}
$$

The model we have just specified has nine parameters, which make the analysis difficult. To reduce the number of parameters, we make the following transformations: $X=f n, Y=\alpha c$, and $Z=k \alpha s$. So, system (1) becomes

$$
\begin{aligned}
& \frac{d X}{d t}=X(p-Y-X), \\
& \frac{d Y}{d t}=Z-(q-X) Y,
\end{aligned}
$$

$$
\frac{d Z}{d t}=-a X Z+a(d+Y) X Y-h Z+\gamma(t),
$$

with initial data: $X(0)>0, Y(0)>0, Z(0)>0$,

where $p=b_{0}-d_{0}, q=r+m+b_{0}, a=k / f, d=r+d_{0}$, and $\gamma(t)=k \alpha u(t)$.

Therefore, from (2), we have

$$
Y(t)<p, \quad \forall t \geq 0[\because Y(t)=\alpha c(t)] .
$$

Theorem 1. Each component of the solution of system (3) subject to initial conditions (4) is positive and bounded.

Proof. From the first equation of the system (3) we get

$$
X(t)=X(0) \exp \left\{\int_{0}^{t} p-Y(s)-X(s) d s\right\}>0 .
$$

From the second equation of (3), we get

$$
\begin{aligned}
\frac{d Y}{d t} & \geq-(q-X) Y \Longrightarrow Y(t) \\
& \geq Y(0) \exp \left\{-\int_{0}^{t}(q-X(s)) d s\right\}>0 .
\end{aligned}
$$

Similarly, from the third equation of (3), we get

$$
\begin{aligned}
\frac{d Z}{d t} & \geq-(a X+h) Z \Longrightarrow Z(t) \\
& \geq Z(0) \exp \left\{-\int_{0}^{t}(a X(s)+h) d s\right\}>0 .
\end{aligned}
$$

Therefore, $X(t)>0, Y(t)>0$, and $Z(t)>0$, for all $t \geq 0$. 
From the first equation of (3), we have

$$
\frac{d X}{d t}=X(p-Y-X) \leq p X\left(1-\frac{X}{p}\right) .
$$

By a standard comparison theorem and by (5), we have,

$$
\limsup _{t \rightarrow \infty} X(t) \leq p, \quad Y(t)<p, \quad \forall t \geq 0 .
$$

From the third equation of (3), we have

$$
\begin{aligned}
\frac{d Z}{d t} & \leq a(d+p) p^{2}-h Z+\gamma^{M} \Longrightarrow \limsup _{t \rightarrow \infty} Z(t) \\
& \leq \frac{a(d+p) p^{2}+\gamma^{M}}{h} ; \quad \gamma^{M}=\limsup _{t \rightarrow \infty} \gamma(t) .
\end{aligned}
$$

So, we conclude that each component of the solution of system (3) subject to (4) is positive and bounded for all $t \geq 0$. This completes the proof.

\section{Stability Behaviour of the Model}

\subsection{Case I: Zero Exogenous Input $(\gamma(t)=0)$}

Theorem 2. If $\gamma(t)=0$, then the model (3) has nonnegative equilibria $E_{0}(0,0,0)$ which is unstable and $E_{1}(p, 0,0)$ which is locally asymptotically stable. The interior equilibrium $E^{*}\left(X^{*}, Y^{*}, Z^{*}\right)$ is not feasible.

Proof. The variational matrix of system (3) at $E_{0}$ is

$$
V\left(E_{0}\right)=\left[\begin{array}{ccc}
p & 0 & 0 \\
0 & -q & 1 \\
0 & 0 & -h
\end{array}\right]
$$

The eigen values are $p,-q$, and $-h$. So, it is obvious that, $E_{0}$ is unstable (hyperbolic saddle).

Now the varitiational matrix of system (3) at $E_{1}$ is

$$
V\left(E_{1}\right)=\left[\begin{array}{ccc}
-p & -p & 0 \\
0 & -(q-p) & 1 \\
0 & a d p & -(a p+h)
\end{array}\right]
$$

The characteristic equation of $V\left(E_{1}\right)$ is $(p+\lambda)\left(\lambda^{2}+B \lambda+\right.$ $C)=0$, where $B=a p+h+q-p=a p+h+d_{0}+r+m>0$ and $C=(a p+h)(q-p)-a p d=a p(q-p-d)+h(q-p)=a p m+$ $h\left(d_{0}+r+m\right)>0$, since $p>0$.

The eigen values are $\lambda_{1}=-p<0$ and $\lambda_{2,3}=(-B \pm$ $\left.\sqrt{B^{2}-4 C}\right) / 2$.

Since $B>0$, and $C>0$, therefore the signs of the real parts of $\lambda_{2}, \lambda_{3}$ are negative. Hence $E_{1}$ is locally asymptotically stable.

It is noted here that the other equilibrium point $\left(X^{*}\right.$, $Y^{*}$, and $Z^{*}$ ) is not feasible, since

$$
X^{*}=\frac{h q}{h-a m}, \quad Y^{*}=\frac{-h\left(r+m+d_{0}\right)-a p m}{h-a m}
$$

are opposite in sign since $p>0$, by (2).

This completes the proof.
3.2. Case II: Nonzero Exogenous Input $(\gamma(t)=Q>0)$. When $(\gamma(t)=Q>0$, a constant), the model (3) has two nonnegative equilibria, $E_{2}(0, Q / h q, Q / h)$ and $E^{*}\left(X^{*}, Y^{*}, Z^{*}\right)$. The variational matrix of system (3) at $E_{2}$ is given by

$$
V\left(E_{2}\right)=\left[\begin{array}{ccc}
p-\frac{Q}{h q} & 0 & 0 \\
\frac{Q}{h q} & -q & 1 \\
-\frac{a Q}{h}+\frac{a Q}{h q}\left(d+\frac{Q}{h q}\right) & 0 & -h
\end{array}\right] .
$$

The characteristic equation of $V\left(E_{2}\right)$ is $(p-(Q / h q)-\lambda)(q+$ $\lambda)(h+\lambda)=0$.

So, $E_{2}$ is locally asymptotically stable if and only if $h p q<$ $Q$ and if $h p q>Q$, and then $E_{2}$ becomes unstable.

The interior equilibrium point $E^{*}\left(X^{*}, Y^{*}, Z^{*}\right)$ of system (3) is given by

$$
\begin{gathered}
X^{*}=\frac{A-h q+p(a m-h)}{2(a m-h)}, \\
Y^{*}=\frac{p(a m-h)+h q-A}{2(a m-h)}, \\
Z^{*}=\frac{\{p(a m-h)+h q-A\}\{(a m-h)(2 q-p)+h q-A\}}{4(a m-h)^{2}},
\end{gathered}
$$

where

$$
\begin{array}{r}
A=\sqrt{\{h q-p(a m-h)\}^{2}+4(a m-h)(h p q-Q)}, \\
m=(q-p-d) .
\end{array}
$$

It can be provided that the unique interior equilibrium point $E^{*}\left(X^{*}, Y^{*}, Z^{*}\right)$ of system (3) exists if and only if the following two conditions are satisfied:

$$
\text { (i) } a m>h, \quad \text { (ii) } h p q>Q \text {. }
$$

Summarizing the above analysis we come to the following theorem.

Theorem 3. If $\gamma(t)=Q>0$, then the equilibrium point $E_{2}(0$, $Q / h q, Q / h)$ of the system (3) is locally asymptotically stable if and only if $h p q<Q$ and the unique interior equilibrium point $E^{*}\left(X^{*}, Y^{*}, Z^{*}\right)$ of system (3) exists if and only if the following two conditions are satisfied:

$$
\text { (i) } a m>h, \quad \text { (ii) } h p q>Q, \quad m=(q-p-d) \text {. }
$$

3.3. Local Stability of $E^{*}\left(X^{*}, Y^{*}, Z^{*}\right)$. The variational matrix at $E^{*}$ is given by

$$
V\left(E^{*}\right)=\left[\begin{array}{ccc}
m_{11} & m_{12} & 0 \\
m_{21} & m_{22} & m_{23} \\
m_{31} & m_{32} & m_{33}
\end{array}\right]
$$


where

$$
\begin{gathered}
m_{11}=-X^{*}, \quad m_{12}=-X^{*}, \\
m_{21}=Y^{*}, \quad m_{22}=-q+X^{*}, \\
m_{23}=1, \quad m_{31}=-a Z^{*}+a Y^{*}\left(d+Y^{*}\right), \\
m_{32}=a X^{*}\left(d+2 Y^{*}\right), \quad m_{33}=-\left(a X^{*}+h\right) .
\end{gathered}
$$

The characteristic equation is

$$
\lambda^{3}+A_{1} \lambda^{2}+A_{2} \lambda+A_{3}=0,
$$

where

$$
\begin{aligned}
A_{1}= & -m_{11}-m_{22}-m_{33}=-\operatorname{tr}\left[V\left(E^{*}\right)\right] \\
A_{2}=m_{11} m_{22}+ & m_{11} m_{33}+m_{22} m_{33}-m_{23} m_{32}-m_{12} m_{21}, \\
A_{3}= & -\operatorname{det}\left[V\left(E^{*}\right)\right] \\
= & m_{11} m_{23} m_{32}+m_{12} m_{21} m_{33} \\
& -m_{11} m_{22} m_{33}-m_{12} m_{23} m_{31} .
\end{aligned}
$$

By the Routh-Hurwitz criterion [28-30], it follows that all eigenvalues of characteristic equation have negative real part if and only if

$$
A_{1}>0, \quad A_{3}>0, \quad A_{1} A_{2}-A_{3}>0 .
$$

Therefore, we come to the following theorem.

Theorem 4. $E^{*}$ is locally asymptotically stable if and only if the inequalities (24) are satisfied.

Furthermore, the conditions arising through Hopf bifurcation cannot be derived explicitly in terms of system parameters. Let us assume that $\mu$ stands for any parameter involved with the model system (3). If there exists a critical magnitude $\mu=\mu^{*}$ such that $A_{1}\left(\mu^{*}\right)>0, A_{3}\left(\mu^{*}\right)>0,\left(A_{1} A_{2}-A_{3}\right)_{\mu=\mu_{*}}=$ 0 , and $(d / d \mu)\left(A_{1} A_{2}-A_{3}\right)_{\mu=\mu_{*}} \neq 0$, then $E^{*}$ undergoes a Hopf bifurcation at $\mu=\mu^{*}$ (Liu’s criterion) [28-30].

3.4. Global Stability of $E^{*} . E^{*}$ is not always globally asymptotically stable. The conditions which guarantee the global stability of $E^{*}$ are stated in the following theorem.

Theorem 5. Since $X(t), Y(t)$, and $Z(t)$ are bounded, there exist positive constants $m_{i}, M_{i}(i=1,2,3)$ such that $m_{1} \leq X(t) \leq$ $M_{1}, m_{2} \leq Y(t) \leq M_{2}$, and $m_{3} \leq Z(t) \leq M_{3}$. If the following inequalities hold:

$$
\begin{gathered}
\left(M_{2}-1\right)^{2}<\left(q-X^{*}\right), \\
\left\{a M_{2}\left(d+M_{2}\right)-a m_{3}\right\}^{2}<\left(a X^{*}+h\right), \\
\left(1+a d X^{*}+2 a X^{*} M_{2}\right)^{2}<\left(q-X^{*}\right)\left(h+a X^{*}\right),
\end{gathered}
$$

then $E^{*}$ is globally asymptotically stable.
Proof. We consider the following positive definite function which has a strict minimum at $E^{*}$ :

$L(X, Y, Z)$

$$
=\left(X-X^{*}-X^{*} \ln \frac{X}{X^{*}}\right)+\frac{1}{2}\left(Y-Y^{*}\right)^{2}+\frac{1}{2}\left(Z-Z^{*}\right)^{2} .
$$

Differentiating both sides with respect to $t$ along the solution of (3), we get (after some simple calculations)

$$
\begin{aligned}
\frac{d L}{d t}= & -\left(X-X^{*}\right)^{2}+(Y-1)\left(X-X^{*}\right)\left(Y-Y^{*}\right) \\
& -\left(q-X^{*}\right)\left(Y-Y^{*}\right)^{2} \\
& +(a Y(d+Y)-a Z)\left(X-X^{*}\right)\left(Z-Z^{*}\right) \\
& -\left(a X^{*}+h\right)\left(Z-Z^{*}\right)^{2} \\
& +\left(1+a d X^{*}+a X^{*}\left(Y+Y^{*}\right)\right)\left(Y-Y^{*}\right)\left(Z-Z^{*}\right) \\
\leq & -\left(X-X^{*}\right)^{2}+\left(M_{2}-1\right)\left(X-X^{*}\right)\left(Y-Y^{*}\right) \\
& -\left(q-X^{*}\right)\left(Y-Y^{*}\right)^{2}-\left(a X^{*}+h\right)\left(Z-Z^{*}\right)^{2} \\
& +\left(a M_{2}\left(d+M_{2}\right)-a m_{3}\right)\left(X-X^{*}\right)\left(Z-Z^{*}\right) \\
& +\left(1+a d X^{*}+2 a M_{2} X^{*}\right)\left(Y-Y^{*}\right)\left(Z-Z^{*}\right) \\
= & -a_{11}\left(X-X^{*}\right)^{2}-a_{22}\left(Y-Y^{*}\right)^{2}-a_{33}\left(Z-Z^{*}\right)^{2} \\
& +a_{12}\left(X-X^{*}\right)\left(Y-Y^{*}\right)+a_{13}\left(X-X^{*}\right)\left(Z-Z^{*}\right) \\
& +a_{23}\left(Y-Y^{*}\right)\left(Z-Z^{*}\right),
\end{aligned}
$$

where

$$
\begin{gathered}
a_{11}=1, \quad a_{22}=q-X^{*}, \quad a_{33}=a X^{*}+h, \\
a_{12}=M_{2}-1, \quad a_{13}=\left\{a M_{2}\left(d+M_{2}\right)-a m_{3}\right\}, \\
a_{23}=1+a d X^{*}+2 a M_{2} X^{*} .
\end{gathered}
$$

Now, sufficient conditions for $d L / d t$ to be negative definite are

$$
a_{12}^{2}-a_{11} a_{22}<0, \quad a_{13}^{2}-a_{11} a_{33}<0, \quad a_{23}^{2}-a_{22} a_{33}<0,
$$

that is,

$$
\begin{gathered}
\left(M_{2}-1\right)^{2}<\left(q-X^{*}\right), \\
\left\{a M_{2}\left(d+M_{2}\right)-a m_{3}\right\}^{2}<\left(a X^{*}+h\right), \\
\left(1+a d X^{*}+2 a X^{*} M_{2}\right)^{2}<\left(q-X^{*}\right)\left(h+a X^{*}\right) .
\end{gathered}
$$

Hence $L$ is a Lyapunov function and so $E^{*}$ is globally asymptotically stable [28-30]. This competes the proof. 


\section{Model with Discrete Delay}

It is already mentioned that time delay is an important factor in biological system. It is also reasonable to assume that the effect of the environmental toxicant on the population growth will not be instantaneous, but mediated by some discrete time lag $\tau$ required for incubation. So, we modify system (3) and (4) as follows:

$$
\begin{gathered}
\frac{d X}{d t}=X(p-Y-X) \\
\frac{d Y}{d t}=Z(t-\tau)-(q-X) Y \\
\frac{d Z}{d t}=-a X Z+a(d+Y) X Y-h Z+Q
\end{gathered}
$$

with initial conditions

$$
\begin{array}{r}
X(0)>0, \quad Y(0)>0, \quad Z(\vartheta)=\varphi(\vartheta), \\
\text { such that } \varphi(\vartheta) \geq 0, \quad \forall \vartheta \in[-\tau, 0],
\end{array}
$$

where $\varphi(\vartheta)$ is a nonnegative continuous functions on $\vartheta \epsilon$ $[-\tau, 0]$. For a biological meaning, we further assume that $\varphi(0)>0$.

All parameters are the same as in system (3) except that the positive constant $\tau$ represents the activation period or reaction time of the toxicant in the population and $\gamma(t)=Q(>$ $0)$ is a constant.

The system (31) has the same equilibria as in art.3.2. The main purpose of this section is to study the stability behaviour of $E^{*}\left(X^{*}, Y^{*}\right.$, and $\left.Z^{*}\right)$ in the presence of discrete delay $(\tau>0)$. We linearize system (31) by using the following transformations:

$$
\begin{gathered}
X(t)=X^{*}+u(t), \quad Y(t)=Y^{*}+v(t), \\
Z(t)=Z^{*}+w(t) .
\end{gathered}
$$

Then transformed linear system is given by

$$
\frac{d U}{d t}=A U(t)+B U(t-\tau),
$$

where

$$
\begin{gathered}
U(t)=\left[\begin{array}{lll}
u & v & w
\end{array}\right]^{T}, \quad A=\left(a_{i j}\right)_{3 \times 3}, \quad B=\left(b_{i j}\right)_{3 \times 3}, \\
a_{11}=-X^{*}, \quad a_{12}=-X^{*}, \\
a_{21}=Y^{*}, \quad a_{22}=-q+X^{*}, \\
a_{31}=-a Z^{*}+a d Y^{*}+a\left(Y^{*}\right)^{2}, \\
a_{32}=a d X^{*}+2 a X^{*} Y^{*}, \\
a_{33}=-\left(a X^{*}+h\right) \text { and all other } a_{i j}=0 ; \\
b_{23}=1 \text { and all other } b_{i j}=0 .
\end{gathered}
$$

Let us take the solution of the system (34) as $u(t)=\rho_{1} e^{\lambda t}$, $v(t)=\rho_{2} e^{\lambda t}, w(t)=\rho_{3} e^{\lambda t}$. This leads to the following characteristic equation:

$$
\lambda^{3}+a_{1} \lambda^{2}+a_{2} \lambda+a_{3}+\left(a_{4} \lambda+a_{5}\right) e^{-\lambda \tau}=0,
$$

where

$$
\begin{gathered}
a_{1}=-a_{11}-a_{22}-a_{33}, \\
a_{2}=a_{11} a_{22}+a_{11} a_{33}+a_{22} a_{33}-a_{12} a_{21}, \\
a_{3}=a_{12} a_{21} a_{33}-a_{11} a_{22} a_{33}, \\
a_{4}=-a_{32} b_{23}, \\
a_{5}=a_{11} a_{32} b_{23}-a_{12} a_{31} b_{23} .
\end{gathered}
$$

A necessary condition for a stability change of $E^{*}$ is that the characteristic equation should have purely imaginary solutions. In order to see the delay induced instability, let us consider $\tau$ as bifurcation parameter and assume a purely imaginary solution of (3) in the form $\lambda=i \omega(\omega \neq 0)$. Therefore, substituting $\lambda=i \omega$ in (37) and separating real and imaginary parts, we get

$$
\begin{aligned}
& a_{1} \omega^{2}-a_{3}=a_{5} \cos (\omega \tau)+a_{4} \omega \sin (\omega \tau), \\
& -\omega^{3}+a_{2} \omega=a_{5} \sin (\omega \tau)-a_{4} \omega \cos (\omega \tau) .
\end{aligned}
$$

Eliminating $\tau$ by squaring and adding (38), we get the equation for determining $\omega$ as

$$
\omega^{6}+d_{1} \omega^{4}+d_{2} \omega^{2}+d_{3}=0,
$$

where

$$
\begin{gathered}
d_{1}=a_{1}^{2}-2 a_{2}, \\
d_{2}=a_{2}^{2}-2 a_{1} a_{3}-a_{4}^{2}, \\
d_{3}=a_{3}^{2}-a_{5}^{2} .
\end{gathered}
$$

Substituting $\omega^{2}=\sigma$ in (40), we get a cubic equation given by

$$
\Phi(\sigma) \equiv \sigma^{3}+d_{1} \sigma^{2}+d_{2} \sigma+d_{3}=0
$$

Now, from (38), we get

$$
\begin{array}{r}
\tau_{k}=\frac{1}{\omega_{0}} \arccos \left(\frac{a_{4} \omega_{0}^{4}+\left(a_{1} a_{5}-a_{2} a_{4}\right) \omega_{0}^{2}-a_{3} a_{5}}{a_{5}^{2}+a_{4} \omega_{0}^{2}}\right)+\frac{2 k \pi}{\omega_{0}}, \\
k=0,1,2, \ldots
\end{array}
$$

Thus when $\tau=\tau_{k}$, the characteristic equation (37) has a pair of purely imaginary roots $\pm i \omega_{0}$. Let $\lambda(\tau)=\xi(\tau)+i \omega(\tau)$ be a root of (37) such that the following two conditions hold:

$$
\xi\left(\tau_{k}\right)=0, \quad \omega\left(\tau_{k}\right)=\omega_{0}
$$


Differentiating both sides of (37) with respect to $\tau$, we get

$$
\begin{aligned}
&\left(\frac{d \lambda}{d \tau}\right)^{-1}= \frac{3 \lambda^{2}+2 a_{1} \lambda+a_{2}}{\left(a_{4} \lambda^{2}+a_{5} \lambda\right) e^{-\lambda \tau}}+\frac{a_{4}}{a_{4} \lambda^{2}+a_{5} \lambda}-\frac{\tau}{\lambda} . \\
& \therefore \operatorname{Re}\left(\frac{d \lambda}{d \tau}\right)_{\lambda=i \omega_{0}}^{-1} \\
&=\frac{1}{\omega_{0}\left(a_{4}^{2} \omega_{0}^{2}+a_{5}^{2}\right)} \\
& \quad \times\left[\left(a_{2} a_{5}-3 \omega_{0}^{2} a_{5}+2 a_{1} a_{4} \omega_{0}^{2}\right) \sin \left(\omega_{0} \tau\right)\right. \\
& \quad+\left(3 a_{4} \omega_{0}^{3}+2 a_{1} a_{5} \omega_{0}-a_{2} a_{4} \omega_{0}\right) \cos \left(\omega_{0} \tau\right) \\
&=\frac{\left.-a_{4}^{2} \omega_{0}\right]}{\left.(d \vartheta(\sigma) / d \sigma]_{\sigma=\omega_{0}^{2}}^{2} \omega_{0}^{2}+a_{5}^{2}\right)} .
\end{aligned}
$$

Now, if $\sigma=\omega_{0}^{2}$ is the first positive root of (41) and hence $\sigma=\omega_{0}(>0)$ is the first positive root of (40), and then

$$
\operatorname{Re}\left(\frac{d \lambda}{d \tau}\right)_{\lambda=i \omega_{0}}^{-1}>0 \quad \text { as }\left[\frac{d \vartheta(\sigma)}{d \sigma}\right]_{\sigma=\omega_{0}^{2}}>0 .
$$

Thus, the transversality condition is satisfied and the steady state becomes unstable when $\tau>\tau_{*}$. Moreover, a Hopf bifurcation [28-30] occurs when $\tau$ passes through the critical value $\tau_{*}$, where $\tau_{*}$ is the smallest positive value of $\tau_{k}$ given in (42).

We notice that, in (41), $\Phi$ is continuous everywhere with $\Phi(0)<0$ and $\Phi(\sigma) \rightarrow \infty$ as $\sigma \rightarrow \infty$. Therefore, the cubic equation (41) always has at least one positive root. Consequently the stability criteria of the system for $\tau=0$ will not necessarily ensure the stability of the system for $\tau \neq 0$. In the following theorem, we have given a criterion for switching the stability behaviour of $E^{*}$.

Theorem 6 (see [28-30]). If $E^{*}$ exists with the conditions (24) and $\sigma_{0}=\omega_{0}^{2}$ be a positive root of $(41)$, then there exists a $\tau=\tau_{*}$ such that

(i) $E^{*}$ is locally asymptotically stable for $0 \leq \tau<\tau_{*}$,

(ii) $E^{*}$ is unstable for $\tau>\tau_{*}$,

(iii) the system (31) undergoes a Hopf-bifurcation around $E^{*}$ at $\tau=\tau_{*}=\min g\left(\omega_{0}\right)$,

where $g\left(\omega_{0}\right)=\frac{1}{\omega_{0}} \arccos$

$$
\times\left(\frac{a_{4} \omega_{0}^{4}+\left(a_{1} a_{5}-a_{2} a_{4}\right) \omega_{0}^{2}-a_{3} a_{5}}{a_{5}^{2}+a_{4} \omega_{0}^{2}}\right)
$$

and the minimum is taken over all positive $\omega_{0}$ such that $\omega_{0}^{2}$ is a solution of (41).

\section{Estimation of the Length of Delay to Preserve Stability}

We linearize the system (31) about its interior equilibrium $E^{*}\left(X^{*}, Y^{*}\right.$, and $\left.Z^{*}\right)$ and get

$$
\begin{gathered}
\frac{d x_{1}}{d t}=a_{11} x_{1}+a_{12} y_{1} \\
\frac{d y_{1}}{d t}=a_{21} x_{1}+a_{22} y_{1}+b_{23} z_{1}(t-\tau) \\
\frac{d z_{1}}{d t}=a_{31} x_{1}+a_{32} y_{1}+a_{33} z_{1}
\end{gathered}
$$

where

$$
\begin{gathered}
X(t)=x_{1}(t)+X^{*}, \quad Y(t)=y_{1}(t)+Y^{*}, \\
Z(t)=z_{1}(t)+Z^{*} .
\end{gathered}
$$

Taking Laplace transform of the system (47), we get

$$
\begin{gathered}
\left(s-a_{11}\right) \overline{x_{1}}(s)=a_{12} \overline{y_{1}}(s)+x_{1}(0) \\
\left(s-a_{22}\right) \overline{y_{1}}(s) \\
=a_{21} \overline{x_{1}}(s)+b_{23} e^{-s \tau} \overline{z_{1}}(s)+b_{23} e^{-s \tau} k_{1}(s)+y_{1}(0) \\
\left(s-a_{33}\right) \overline{z_{1}}(s)=a_{31} \overline{x_{1}}(s)+a_{32} \overline{y_{1}}(s)+z_{1}(0),
\end{gathered}
$$

where

$$
k_{1}(s)=\int_{-\tau}^{0} e^{-s t} z_{1}(t) d t
$$

and $\overline{x_{1}}(s), \overline{y_{1}}(s)$, and $\overline{z_{1}}(s)$ are the Laplace transform of $x_{1}(t)$, $y_{1}(t)$ and $z_{1}(t)$, respectively.

Now, we will use the "Nyquist theorem" [21] which states that if $s$ is the arc length along a curve encircling the right half of the plane, then a curve $\Gamma(s)$ will encircle the origin a number of times equal to the difference between the number of poles and the number of zeros of $\Gamma(s)$ in the right half of the plane. Using "Nyquist theorem" $[21,31]$, it can be shown that the conditions for local asymptotic stability of $E^{*}\left(X^{*}, Y^{*}, Z^{*}\right)$ are given by [32]

$$
\begin{aligned}
& \operatorname{Im} H\left(i \omega_{0}\right)>0, \\
& \operatorname{Re} H\left(i \omega_{0}\right)=0,
\end{aligned}
$$

where $H(s)=s^{3}+a_{1} s^{2}+a_{2} s+a_{3}+\left(a_{4} s+a_{5}\right) e^{-s \tau}$ and $\omega_{0}$ is the smallest positive root of (52).

We have already shown that $E^{*}\left(X^{*}, Y^{*}, Z^{*}\right)$ is stable in absence of delay (provided that inequalities given in (24) are satisfied). Hence, by continuity, all eigenvalues will continue to have negative real parts for sufficiently small $\tau>0$ provided that one can guarantee that no eigenvalue with positive real part bifurcates from infinity as $\tau$ increases from zero. This can be proved by using Butler's lemma [32].

In this case, conditions (51) and (52) give

$$
\begin{aligned}
& a_{2} \omega_{0}-\omega_{0}^{3}>a_{5} \sin \left(\omega_{0} \tau\right)-a_{4} \omega_{0} \cos \left(\omega_{0} \tau\right), \\
& a_{3}-a_{1} \omega_{0}^{2}=-a_{5} \cos \left(\omega_{0} \tau\right)-a_{4} \omega_{0} \sin \left(\omega_{0} \tau\right) .
\end{aligned}
$$


Now, (53) and (54), if satisfied simultaneously, are sufficient conditions to guarantee stability. We will utilize them to get an estimate on the length of delay. Our aim is to find an upper bound $\omega_{+}$on $\omega_{0}$, independent of $\tau$ so that (53) holds for all values of $\omega, 0 \leq \omega \leq \omega_{+}$and hence in particular at $\omega=\omega_{0}$.

We rewrite (54) as

$$
a_{1} \omega_{0}^{2}=a_{3}+a_{5} \cos \left(\omega_{0} \tau\right)+a_{4} \omega_{0} \sin \left(\omega_{0} \tau\right) .
$$

Maximizing $a_{3}+a_{5} \cos \left(\omega_{0} \tau\right)+a_{4} \omega_{0} \sin \left(\omega_{0} \tau\right)$ subject to $\left|\sin \left(\omega_{0} \tau\right)\right| \leq 1,\left|\cos \left(\omega_{0} \tau\right)\right| \leq 1$, we obtain

$$
a_{1} \omega_{0}^{2} \leq a_{3}+\left|a_{5}\right|+\left|a_{4}\right| \omega_{0}
$$

Hence, if

$$
\omega_{+}=\frac{1}{2 a_{1}}\left[\left|a_{4}\right|+\sqrt{a_{4}^{2}+4 a_{1}\left(a_{3}+\left|a_{5}\right|\right)}\right],
$$

then clearly from (56) and (57), we have $\omega_{0} \leq \omega_{+}$.

From the inequality (53), we get

$$
\omega_{0}^{2}<a_{2}+a_{4} \cos \left(\omega_{0} \tau\right)-\frac{a_{5}}{\omega_{0}} \sin \left(\omega_{0} \tau\right)
$$

As $E^{*}\left(X^{*}, Y^{*}, Z^{*}\right)$ is locally asymptotically stable for $\tau=$ 0 (provided that inequalities given in (24) are satisfied), for sufficiently small $\tau>0$, (58) will continue to hold. Substituting (55) into (58) and rearranging, we get

$$
\begin{gathered}
\left(a_{1} a_{4}-a_{5}\right)\left\{1-\cos \left(\omega_{0} \tau\right)\right\}+\left(a_{4} \omega_{0}+\frac{a_{1} a_{5}}{\omega_{0}}\right) \sin \left(\omega_{0} \tau\right) \\
<a_{1} a_{2}+a_{1} a_{4}-a_{3}-a_{5} .
\end{gathered}
$$

Using the bounds we obtain

$$
\begin{gathered}
\left(a_{1} a_{4}-a_{5}\right)\left\{1-\cos \left(\omega_{0} \tau\right)\right\} \\
=\left(a_{1} a_{4}-a_{5}\right) 2 \sin ^{2}\left(\frac{\omega_{0} \tau}{2}\right) \\
\leq \frac{1}{2}\left|a_{1} a_{4}-a_{5}\right| \omega_{+}^{2} \tau^{2}, \\
\left(a_{4} \omega_{0}+\frac{a_{1} a_{5}}{\omega_{0}}\right) \sin \left(\omega_{0} \tau\right) \leq\left(a_{4} \omega_{+}^{2}+a_{1} a_{5}\right) \tau .
\end{gathered}
$$

Now, from (59) and (60), we get

$$
l_{1} \tau^{2}+l_{2} \tau<l_{3}
$$

where

$$
\begin{gathered}
l_{1}=\frac{1}{2}\left|a_{1} a_{4}-a_{5}\right| \omega_{+}^{2}, \\
l_{2}=\left(a_{4} \omega_{+}^{2}+a_{1} a_{5}\right), \\
l_{3}=a_{1} a_{2}+a_{1} a_{4}-a_{3}-a_{5} . \\
\text { Hence, if } \tau_{+}=\frac{1}{2 l_{1}}\left[-l_{2}+\sqrt{l_{2}^{2}+4 l_{1} l_{3}}\right],
\end{gathered}
$$

then stability is preserved for $0 \leq \tau<\tau_{+}$.

Summarizing the previous discussions we come to the following theorem.
Theorem 7. The delayed model (31) and (32) will be locally asymptotically stable at $E^{*}\left(X^{*}, Y^{*}\right.$, and $\left.Z^{*}\right)$ with the conditions $(24)$ if the delay $\tau$ lies within the interval $\left(0, \tau_{+}\right)$where $\tau_{+}$ is given by (63).

\section{Direction of Hopf Bifurcation}

In this section, we will derive the direction of the Hopf bifurcation $[17,21,33]$ and sufficient conditions of the stability of bifurcating periodic solution from the interior equilibrium $E^{*}$ of the system (31) at the critical value $\tau=\tau_{*}$. We will employ the approach of the normal form method and center manifold theorem introduced by Hassard et al. [33].

Let $u_{1}=X-X^{*}, u_{2}=Y-Y^{*}, u_{3}=Z-Z^{*}, \tau=\tau_{*}+$ $\mu, u(t)=\left(u_{1}(t), u_{2}(t), u_{3}(t)\right)^{T}$, and $u_{t}(\theta)=u(t+\theta)$ for $\theta \epsilon$ $\left[-\tau_{*}, 0\right] . \mathscr{C}=C\left(\left[-\tau_{*}, 0\right], \mathbb{R}^{3}\right)$, and we can rewrite the system (31) and (32) as follows:

$$
\dot{u}(t)=L_{\mu}\left(u_{t}\right)+F\left(u_{t}, \mu\right),
$$

where $L_{\mu}: \mathbb{R} \times \mathscr{C} \rightarrow \mathbb{R}^{3}, F: \mathbb{R} \times \mathscr{C} \rightarrow \mathbb{R}^{3}$ are given, respectively, by

$$
L_{\mu}(\phi)=B_{1} \phi(0)+B_{2} \phi\left(-\tau_{*}\right),
$$

$$
\begin{aligned}
& F(\phi, \mu) \\
& \quad=\left[\begin{array}{c}
-\phi_{1}^{2}(0)-\phi_{1}(0) \phi_{2}(0) \\
\phi_{1}(0) \phi_{2}(0) \\
a X^{*} \phi_{2}^{2}(0)+\left(a d+2 a Y^{*}\right) \phi_{1}(0) \phi_{2}(0)-a \phi_{1}(0) \phi_{3}(0)
\end{array}\right],
\end{aligned}
$$

where

$$
\begin{gathered}
B_{1}=\left(\begin{array}{lll}
a_{11} & a_{12} & 0 \\
a_{21} & a_{22} & 0 \\
a_{31} & a_{32} & a_{33}
\end{array}\right), \\
B_{2}=\left(\begin{array}{ccc}
0 & 0 & 0 \\
0 & 0 & b_{23} \\
0 & 0 & 0
\end{array}\right), \\
a_{11}=-X^{*}, \quad X_{21}^{*}=Y^{*}, \\
a_{22}=-q+X^{*}, \quad a_{31}=-a Z^{*}+a d Y^{*}+a\left(Y^{*}\right)^{2}, \\
a_{32}=a d X^{*}+2 a X^{*} Y^{*}, \\
a_{33}=-\left(a X^{*}+h\right) \text { and all other } a_{i j}=0 ; \\
b_{23}=1 \text { and all other } b_{i j}=0 .
\end{gathered}
$$

By the Riesz representation theorem, there exists a matrix function whose components are bounded variation function $\eta(\theta, \mu)$ in $\theta \in\left[-\tau_{*}, 0\right]$ such that

$$
L_{\mu}(\theta)=\int_{-\tau_{*}}^{0} d \eta(\theta, \mu) \phi(\theta), \quad \phi \in \mathscr{C} .
$$

In fact we can choose

$$
\eta(\theta, \mu)=B_{1} \delta(\theta)+B_{2} \delta\left(\theta+\tau_{*}\right),
$$

where $\delta(\theta)$ is the Dirac delta function. 
For $\phi \in \mathscr{C}^{1}\left(\left[-\tau_{*}, 0\right], \mathbb{R}^{3}\right)$, we define

$$
\begin{gathered}
A(\mu) \phi(\theta)= \begin{cases}\frac{d \phi(\theta)}{d \theta}, & \theta \in\left[-\tau_{*}, 0\right) \\
\int_{-\tau_{*}}^{0} d \eta(\theta, \mu) \phi(\theta), & \theta=0,\end{cases} \\
R(\mu) \phi(\theta)= \begin{cases}0, & \theta \in\left[-\tau_{*}, 0\right) \\
F(\mu, \phi), & \theta=0 .\end{cases}
\end{gathered}
$$

Then the system (64) is equivalent to

$$
\dot{u}_{t}=A(\mu) u_{t}+R(\mu) u_{t} .
$$

For $\psi \in \mathscr{C}^{1}\left(\left[0, \tau_{*}\right],\left(\mathbb{R}^{3}\right)^{*}\right)$, where $\left(\mathbb{R}^{3}\right)^{*}$ is the 3-dimensional space of row vectors, the adjoint operator $A^{*}$ of $\mathrm{A}$ is defined as

$$
A^{*}(\mu) \psi(s)= \begin{cases}-\frac{d \psi(s)}{d s}, & s \in\left(0, \tau_{*}\right] \\ \int_{-\tau_{*}}^{0} d \eta^{T}(s, \mu) \psi(-s), & s=0 .\end{cases}
$$

For $\phi \in\left[-\tau_{*}, 0\right)$ and $\psi \in\left[0, \tau_{*}\right]$, we define a bilinear inner product taking the first argument to be conjugate linear and the second to be linear of the form

$$
\langle\psi, \phi\rangle=\bar{\psi}(0) \phi(0)-\int_{\theta=-\tau_{*}}^{0} \int_{\xi=0}^{\theta} \bar{\psi}(\xi-\theta) d \eta(\theta) \phi(\xi) d \xi
$$

where $\eta(\theta)=\eta(\theta, 0)$. It is easy to verify that $A(0)$ and $A^{*}$ are a pair of adjoint operators.

From the discussions in the previous section, we know that $\pm i \omega_{0}$ are the eigenvalues of $A(0)$. Hence, the eigenvalues of $A^{*}$ are $\mp i \omega_{0}$.

Suppose that $q(\theta)=\left(1, \alpha_{1}, \alpha_{2}\right)^{T} e^{i \omega_{0} \theta}$ is eigenvector of $A(0)$ corresponding to $i \omega_{0}$, and then we have

$$
A(0) q(\theta)=i \omega_{0} q(\theta) \text {. }
$$

It follows from (67), (68), and the definition of $A(0)$ that

$$
\left[\left(B_{1}+B_{2} e^{-i \omega_{0} \tau_{*}}\right)-i \omega_{0} I\right] q(0)=0
$$

where $I$ is identity matrix of order $3 \times 3$, that is,

$$
\left(\begin{array}{ccc}
a_{11}-i \omega_{0} & a_{12} & 0 \\
a_{21} & a_{22}-i \omega_{0} & b_{23} e^{-i \omega_{0} \tau_{*}} \\
a_{31} & a_{32} & a_{33}-i \omega_{0}
\end{array}\right) q(0)=\left(\begin{array}{l}
0 \\
0 \\
0
\end{array}\right)
$$

Solving (76), we have $q(0)=\left(1, \alpha_{1}, \alpha_{2}\right)^{T}$, where

$$
\begin{gathered}
\alpha_{1}=\frac{i \omega_{0}-a_{11}}{a_{12}} \\
\alpha_{2}=-\frac{a_{12} a_{21}+\left(a_{22}-i \omega_{0}\right)\left(i \omega_{0}-a_{11}\right)}{a_{12} b_{23} e^{-i \omega_{0} \tau_{*}}} .
\end{gathered}
$$

Now, suppose that $q^{*}(s)=B\left(1, \alpha_{1}^{*}, \alpha_{2}^{*}\right) e^{i \omega_{0} s}$ is the eigenvector of $A^{*}$ corresponding to $-i \omega_{0}$, and then we have

$$
A^{*}(0) q^{*}(s)=-i \omega_{0} q^{*}(s) .
$$

It follows from (67), (68), and the definition of $A^{*}$ that,

$$
\left[\left(B_{1}^{T}+B_{2}^{T} e^{i \omega_{0} \tau_{*}}\right)+i \omega_{0} I\right]\left(q^{*}(0)\right)^{T}=0,
$$

where $I$ is identity matrix of order $3 \times 3$, that is,

$$
\left(\begin{array}{ccc}
a_{11}+i \omega_{0} & a_{21} & a_{31} \\
a_{12} & a_{22}+i \omega_{0} & a_{32} \\
0 & b_{23} e^{i \omega_{0} \tau_{*}} & a_{33}+i \omega_{0}
\end{array}\right)\left(q^{*}(0)\right)^{T}=\left(\begin{array}{l}
0 \\
0 \\
0
\end{array}\right) .
$$

Solving (80), we have $q^{*}(0)=B\left(1, \alpha_{1}^{*}, \alpha_{2}^{*}\right)$, where

$$
\begin{aligned}
& \alpha_{1}^{*}=-\frac{\left(a_{11}+i \omega_{0}\right)\left(a_{33}+i \omega_{0}\right)}{a_{21}\left(a_{33}+i \omega_{0}\right)-a_{31} b_{23} e^{i \omega_{0} \tau_{*}}}, \\
& \alpha_{2}^{*}=\frac{\left(a_{11}+i \omega_{0}\right) b_{23} e^{i \omega_{0} \tau_{*}}}{a_{21}\left(a_{33}+i \omega_{0}\right)-a_{31} b_{23} e^{i \omega_{0} \tau_{*}}} .
\end{aligned}
$$

Now,

$$
\begin{aligned}
& \left\langle q^{*}(s), q(\theta)\right\rangle \\
& =\bar{B}\left\{\left(1, \bar{\alpha}_{1}^{*}, \bar{\alpha}_{2}^{*}\right)\left(1, \alpha_{1}, \alpha_{2}\right)^{T}\right. \\
& \quad-\int_{\theta=-\tau_{*}}^{0} \int_{\xi=0}^{\theta}\left(1, \bar{\alpha}_{1}^{*}, \bar{\alpha}_{2}^{*}\right) e^{-i(\xi-\theta) \omega_{0}} d \eta(\theta) \\
& \left.\times \bar{B}\left[1+\alpha_{1} \bar{\alpha}_{1}^{*}+\alpha_{2} \bar{\alpha}_{2}^{*} \alpha_{1}, \alpha_{2}\right)^{T} e^{i \xi \omega_{0}} d \xi\right\} \\
& \left.-\int_{\theta=-\tau_{*}}^{0}\left(1, \bar{\alpha}_{1}^{*}, \bar{\alpha}_{2}^{*}\right) \theta e^{i \omega_{0} \theta} d \eta(\theta)\left(1, \alpha_{1}, \alpha_{2}\right)^{T}\right] \\
& =\bar{B}\left[1+\alpha_{1} \bar{\alpha}_{1}^{*}+\alpha_{2} \bar{\alpha}_{2}^{*}+\tau_{*} e^{-i \omega_{0} \tau_{*}}\left(\alpha_{2} \bar{\alpha}_{1}^{*} b_{23}\right)\right] .
\end{aligned}
$$

Thus in order to assume $\left\langle q^{*}(s), q(\theta)\right\rangle=1$, we can choose

$$
B=\frac{1}{1+\alpha_{1} \bar{\alpha}_{1}^{*}+\alpha_{2} \bar{\alpha}_{2}^{*}+\tau_{*} e^{-i \omega_{0} \tau_{*}}\left(\alpha_{2} \bar{\alpha}_{1}^{*}\right)} .
$$

Also since $\langle\psi, A \phi\rangle=\left\langle A^{*} \psi, \phi\right\rangle$, we have

$$
\begin{aligned}
-i \omega_{0}\left\langle q^{*}, \bar{q}\right\rangle & =\left\langle q^{*}, A \bar{q}\right\rangle, \\
& =\left\langle A^{*} q^{*}, \bar{q}\right\rangle, \\
& =\left\langle-i \omega_{0} q^{*}, \bar{q}\right\rangle, \\
& =i \omega_{0}\left\langle q^{*}, \bar{q}\right\rangle .
\end{aligned}
$$

Therefore, $\left\langle q^{*}, \bar{q}\right\rangle=0$. 
Now, we first compute the coordinates to describe the center manifold $C_{0}$ at $\mu=0$. Let $u_{t}$ be the solution of (64) when $\mu=0$. Define

$$
\begin{gathered}
z(t)=\left\langle q^{*}, u_{t}\right\rangle, \\
W(t, \theta)=u_{t}-z(t) q(\theta)-\bar{z}(t) \bar{q}(\theta) \\
=u_{t}(\theta)-2 \operatorname{Re}\{z(t) q(\theta)\} .
\end{gathered}
$$

On the center manifold $C_{0}$, we have

$$
W(z, \bar{z}, \theta)=W_{20}(\theta) \frac{z^{2}}{2}+W_{11}(\theta) z \bar{z}+W_{02}(\theta) \frac{\bar{z}^{2}}{2}+\cdots
$$

where $z$ and $\bar{z}$ are local coordinates of center manifold $C_{0}$ in the direction of $q^{*}$ and $\bar{q}^{*}$. Note that $W$ is real if $u_{t}$ is real. We only consider real solutions.

From (85), we get

$$
\begin{aligned}
\left\langle q^{*}, W\right\rangle & =\left\langle q^{*}, u_{t}-z q-\overline{z q}\right\rangle \\
& =\left\langle q^{*}, u_{t}\right\rangle-z\left\langle q^{*}, q\right\rangle-\bar{z}\left\langle q^{*}, \bar{q}\right\rangle=z-z-0=0 .
\end{aligned}
$$

For the solution $u_{t} \in C_{0}$ of (64) and since $\mu=0$, we have

$$
\begin{aligned}
\dot{z}(t) & =\left\langle q^{*}, \dot{u}_{t}\right\rangle \\
& =\left\langle q^{*}, A(0) u_{t}+R(0) u_{t}\right\rangle \\
& =\left\langle A^{*}(0) q^{*}, u_{t}\right\rangle+\bar{q}^{*}(0) F\left(0, u_{t}\right) \\
& =\left\langle-i \omega_{0} q^{*}, u_{t}\right\rangle+\bar{q}^{*}(0) f_{0}(z, \bar{z}) \\
& =i \omega_{0} z+\bar{q}^{*}(0) f_{0}(z, \bar{z}) \\
& =i \omega_{0} z+g(z, \bar{z}),
\end{aligned}
$$

where

$$
\begin{aligned}
g(z, \bar{z}) & =\bar{q}^{*}(0) f_{0}(z, \bar{z}) \\
& =g_{20} \frac{z^{2}}{2}+g_{11} z \bar{z}+g_{02} \frac{\bar{z}^{2}}{2}+g_{21} \frac{z^{2} \bar{z}}{2}+\cdots .
\end{aligned}
$$

Since $u_{t}=W(z, \bar{z}, \theta)+z q+\overline{z q}$, we have

$$
\begin{aligned}
& \left(\begin{array}{l}
u_{1 t}(\theta) \\
u_{2 t}(\theta) \\
u_{3 t}(\theta)
\end{array}\right) \\
& \quad=\left(\begin{array}{l}
W^{(1)}(\theta) \\
W^{(2)}(\theta) \\
W^{(3)}(\theta)
\end{array}\right)+z\left(\begin{array}{c}
1 \\
\alpha_{1} \\
\alpha_{2}
\end{array}\right) e^{i \omega_{0} \theta}+\bar{z}\left(\begin{array}{c}
1 \\
\bar{\alpha}_{1} \\
\bar{\alpha}_{2}
\end{array}\right) e^{-i \omega_{0} \theta} \\
& =\left(\begin{array}{c}
\Gamma_{1} \\
\Gamma_{2} \\
\Gamma_{3}
\end{array}\right)
\end{aligned}
$$

where

$$
\begin{aligned}
\Gamma_{1}= & z e^{i \omega_{0} \theta}+\bar{z} e^{-i \omega_{0} \theta}+W_{20}^{(1)}(\theta) \frac{z^{2}}{2}+W_{11}^{(1)}(\theta) z \bar{z} \\
& +W_{02}^{(1)}(\theta) \frac{\bar{z}^{2}}{2}+o\left(|(z, \bar{z})|^{3}\right), \\
\Gamma_{2}= & \alpha_{1} z e^{i \omega_{0} \theta}+\bar{\alpha}_{1} \bar{z} e^{-i \omega_{0} \theta}+W_{20}^{(2)}(\theta) \frac{z^{2}}{2}+W_{11}^{(2)}(\theta) z \bar{z} \\
& +W_{02}^{(2)}(\theta) \frac{\bar{z}^{2}}{2}+o\left(|z, \bar{z}|^{3}\right), \\
\Gamma_{3}= & \alpha_{2} z e^{i \omega_{0} \theta}+\bar{\alpha}_{2} \bar{z} e^{-i \omega_{0} \theta}+W_{20}^{(3)}(\theta) \frac{z^{2}}{2}+W_{11}^{(3)}(\theta) z \bar{z} \\
& +W_{02}^{(3)}(\theta) \frac{\bar{z}^{2}}{2}+o\left(|z, \bar{z}|^{3}\right) .
\end{aligned}
$$

Then,

$$
\begin{aligned}
u_{1 t}(0)= & z+\bar{z}+W_{20}^{(1)}(0) \frac{z^{2}}{2}+W_{11}^{(1)}(0) z \bar{z} \\
& +W_{02}^{(1)}(0) \frac{\bar{z}^{2}}{2}+o\left(|z, \bar{z}|^{3}\right), \\
u_{2 t}(0)= & \alpha_{1} z+\bar{\alpha}_{1} \bar{z}+W_{20}^{(2)}(0) \frac{z^{2}}{2}+W_{11}^{(2)}(0) z \bar{z} \\
& +W_{02}^{(2)}(0) \frac{\bar{z}^{2}}{2}+o\left(|z, \bar{z}|^{3}\right), \\
u_{3 t}(0)= & \alpha_{2} z+\bar{\alpha}_{2} \bar{z}+W_{20}^{(3)}(0) \frac{z^{2}}{2}+W_{11}^{(3)}(0) z \bar{z} \\
& +W_{02}^{(3)}(0) \frac{\bar{z}^{2}}{2}+o\left(|z, \bar{z}|^{3}\right), \\
u_{1 t}^{2}(0)= & z^{2}+2 z \bar{z}+\bar{z}^{2}+\left[W_{20}^{(1)}(0)+2 W_{11}^{(1)}(0)\right] z^{2} \bar{z} \\
& + \text { h.o.t., } \\
& +\left[\bar{\alpha}_{1} W_{20}^{(2)}(0)+2 \alpha_{1} W_{11}^{(2)}(0)\right] z^{2} \bar{z}+\text { h.o.t., }
\end{aligned}
$$

$$
\begin{aligned}
& u_{1 t}(0) u_{2 t}(0) \\
& =\alpha_{1} z^{2}+\left(\alpha_{1}+\bar{\alpha}_{1}\right) z \bar{z}+\bar{\alpha}_{1} \bar{z}^{2} \\
& +\left[W_{11}^{(1)}(0) \alpha_{1}+\frac{1}{2} W_{20}^{(2)}(0)+\frac{1}{2} W_{20}^{(1)}(0) \bar{\alpha}_{1}\right. \\
& \left.+W_{11}^{(2)}(0)\right] z^{2} \bar{z}+\text { h.o.t. } \\
& \begin{array}{c}
u_{1 t}(0) u_{3 t}(0) \\
=\alpha_{2} z^{2}+\left(\alpha_{2}+\bar{\alpha}_{2}\right) z \bar{z}+\bar{\alpha}_{1} \bar{\alpha}_{2} \bar{z}^{2} \\
+\left[W_{11}^{(1)}(0) \alpha_{2}+\frac{1}{2} W_{20}^{(1)}(0) \bar{\alpha}_{2}+\frac{1}{2} W_{20}^{(3)}(0)\right. \\
\left.+W_{11}^{(3)}(0)\right] z^{2} \bar{z}+\text { h.o.t. }
\end{array}
\end{aligned}
$$


It follows that

$$
f_{0}(z, \bar{z})=\left[\begin{array}{l}
\Delta_{11} z^{2}+\Delta_{12} z \bar{z}+\Delta_{13} \bar{z}^{2}+\Delta_{14} z^{2} \bar{z} \\
\Delta_{21} z^{2}+\Delta_{22} z \bar{z}+\Delta_{23} \bar{z}^{2}+\Delta_{24} z^{2} \bar{z} \\
\Delta_{31} z^{2}+\Delta_{32} z \bar{z}+\Delta_{33} \bar{z}^{2}+\Delta_{34} z^{2} \bar{z}
\end{array}\right]+\cdots,
$$

where

$$
\begin{gathered}
\Delta_{11}=-1-\alpha_{1}, \\
\Delta_{12}=-2-\left(\alpha_{1}+\bar{\alpha}_{1}\right), \\
\Delta_{13}=-1-\bar{\alpha}_{1}, \\
\Delta_{14}=-\left[W_{20}^{(1)}(0)+2 W_{11}^{(1)}(0)+\frac{1}{2} W_{20}^{(1)}(0) \bar{\alpha}_{1}\right. \\
\left.+\frac{1}{2} W_{20}^{(2)}(0)+W_{11}^{(1)}(0) \alpha_{1}+W_{11}^{(2)}(0)\right], \\
\Delta_{21}=\alpha_{1}, \\
\Delta_{22}=\alpha_{1}+\bar{\alpha}_{1}, \\
\Delta_{23}=\bar{\alpha}_{1}, \\
\Delta_{32}=2 a X^{*}\left|\alpha_{10}\right|^{2}+\left(a d+2 a Y^{*}\right)\left(\alpha_{1}+\bar{\alpha}_{1}\right)-a\left(\alpha_{2}+\bar{\alpha}_{2}\right), \\
\Delta_{33}=a X^{*} \bar{\alpha}_{1}^{2}+\left(a d+2 a Y^{*}\right) \bar{\alpha}_{1}-a \bar{\alpha}_{2}, \\
\Delta_{34}, a X^{*} \alpha_{1}^{2}+(0)+W_{11}^{(1)}(0) \alpha_{1}+W_{11}^{(2)}(0), \\
+a\left[X^{*}\left[\bar{\alpha}_{1} W_{20}^{(2)}(0)+2 \alpha_{1} W_{11}^{(2)}(0)\right]+\left(a d+2 a Y^{*}\right)\right. \\
\times\left[W_{11}^{(1)}(0) \alpha_{1}+\frac{1}{2} W_{20}^{(2)}(0)+\frac{1}{2} W_{20}^{(1)}(0) \bar{\alpha}_{1}\right. \\
\left.+W_{11}^{(2)}(0)\right] \\
\left.+W_{11}^{(1)}(0) \alpha_{2}+\frac{1}{2} W_{20}^{(1)}(0) \bar{\alpha}_{2}+\frac{1}{2} W_{20}^{(3)}(0)\right]
\end{gathered}
$$

Since $\bar{q}^{*}(0)=\bar{B}\left(1, \bar{\alpha}_{1}^{*}, \bar{\alpha}_{2}^{*}\right)$, then we obtain

$$
\begin{aligned}
g(z, \bar{z})= & \bar{q}^{*}(0) f_{0}(z, \bar{z}) \\
= & \bar{B}\left(1, \bar{\alpha}_{1}^{*}, \bar{\alpha}_{2}^{*}\right) \\
& \times\left[\begin{array}{c}
\Delta_{11} z^{2}+\Delta_{12} z \bar{z}+\Delta_{13} \bar{z}^{2}+\Delta_{14} z^{2} \bar{z} \\
\Delta_{21} z^{2}+\Delta_{22} z \bar{z}+\Delta_{23} \bar{z}^{2}+\Delta_{24} z^{2} \bar{z} \\
\Delta_{31} z^{2}+\Delta_{32} z \bar{z}+\Delta_{33} \bar{z}^{2}+\Delta_{34} z^{2} \bar{z}
\end{array}\right]+\cdots
\end{aligned}
$$

$$
\begin{aligned}
=\bar{B}[ & \left(\Delta_{11}+\bar{\alpha}_{1}^{*} \Delta_{21}+\bar{\alpha}_{2}^{*} \Delta_{31}\right) z^{2} \\
& +\left(\Delta_{12}+\bar{\alpha}_{1}^{*} \Delta_{22}+\bar{\alpha}_{2}^{*} \Delta_{32}\right) z \bar{z} \\
& +\left(\Delta_{13}+\bar{\alpha}_{1}^{*} \Delta_{23}+\bar{\alpha}_{2}^{*} \Delta_{33}\right) \bar{z}^{2} \\
& \left.+\left(\Delta_{14}+\bar{\alpha}_{1}^{*} \Delta_{24}+\bar{\alpha}_{2}^{*} \Delta_{34}\right) z^{2} \bar{z}\right]+\cdots .
\end{aligned}
$$

The comparison of the coefficients of $z^{2}, z \bar{z}, \bar{z}^{2}, z^{2} \bar{z}$ from (89) and (95) produces

$$
\begin{aligned}
& g_{20}=2 \bar{B}\left(\Delta_{11}+\bar{\alpha}_{1}^{*} \Delta_{21}+\bar{\alpha}_{2}^{*} \Delta_{31}\right) \\
& g_{11}=\bar{B}\left(\Delta_{12}+\bar{\alpha}_{1}^{*} \Delta_{22}+\bar{\alpha}_{2}^{*} \Delta_{32}\right) \\
& g_{02}=2 \bar{B}\left(\Delta_{13}+\bar{\alpha}_{1}^{*} \Delta_{23}+\bar{\alpha}_{2}^{*} \Delta_{33}\right) \\
& g_{21}=2 \bar{B}\left(\Delta_{14}+\bar{\alpha}_{1}^{*} \Delta_{24}+\bar{\alpha}_{2}^{*} \Delta_{34}\right) .
\end{aligned}
$$

Clearly, $g_{21}$ involves the terms $W_{20}, W_{11}$, and we still need to compute $W_{20}, W_{11}$ as

$\dot{W}=\dot{u}_{t}-\dot{z} q-\dot{\bar{z} q}$

$$
= \begin{cases}A(0) W-2 \operatorname{Re}\left(\bar{q}^{*}(0) f_{0}(z, \bar{z}) q(\theta)\right), & \theta \in\left[-\tau_{*} 0\right) \\ A(0) W-2 \operatorname{Re}\left(\bar{q}^{*}(0) f_{0}(z, \bar{z}) q(\theta)\right)+f_{0}(z, \bar{z}), & \theta=0\end{cases}
$$

which is rewritten as

$$
\dot{W}=A(0) W+H(z, \bar{z}, \theta),
$$

where

$$
H(z, \bar{z}, \theta)=H_{20}(\theta) \frac{z^{2}}{2}+H_{11}(\theta) z \bar{z}+H_{02} \frac{\bar{z}^{2}}{2}+\cdots .
$$

On the other hand, on $C_{0}$

$$
\dot{W}=W_{z} \dot{z}+W_{\bar{z}} \dot{\bar{z}} .
$$

We derive the following inequalities from (98)-(100):

$$
\begin{gathered}
\left(A(0)-2 i \omega_{0}\right) W_{20}(\theta)=-H_{20}(\theta) \\
A(0) W_{11}(\theta)=-H_{11}(\theta) .
\end{gathered}
$$

For $\theta \in\left[-\tau_{*}, 0\right)$, it follows from (89), (97), and (98) that $H(z, \bar{z}, \theta)$

$$
\begin{aligned}
= & -2 \operatorname{Re}\left(\bar{q}^{*}(0) f_{0}(z, \bar{z}) q(\theta)\right) \\
= & -2 \operatorname{Re}(g(z, \bar{z}) q(\theta)) \\
= & -g(z, \bar{z}) q(\theta)-\bar{g}(z, \bar{z}) \bar{q}(\theta) \\
= & -\left(g_{20} \frac{z^{2}}{2}+g_{11} z \bar{z}+g_{02} \frac{\bar{z}^{2}}{2}+g_{21} \frac{z^{2} \bar{z}}{2}+\cdots\right) q(\theta) \\
& -\left(\bar{g}_{20} \frac{\bar{z}^{2}}{2}+\bar{g}_{11} z \bar{z}+g_{02} \frac{z^{2}}{2}+\bar{g}_{21} \frac{\bar{z}^{2} z}{2}+\cdots\right) \bar{q}(\theta) .
\end{aligned}
$$


Computing this equation with (99), we get

$$
\begin{aligned}
& H_{20}(\theta)=-g_{20} q(\theta)-\bar{g}_{02} \bar{q}(\theta), \\
& H_{11}(\theta)=-g_{11} q(\theta)-\bar{g}_{11} \bar{q}(\theta) .
\end{aligned}
$$

For $\theta \in\left[-\tau_{*}, 0\right)$, it follows from (69), (101), and (103) that

$$
\begin{aligned}
\dot{W}_{20}(\theta) & =A(0) W_{20}(\theta) \\
& =2 i \omega_{0} W_{20}(\theta)-H_{20}(\theta) \\
& =2 i \omega_{0} W_{20}(\theta)+g_{20} q(\theta)+\bar{g}_{02} \bar{q}(\theta) \\
& =2 i \omega_{0} W_{20}(\theta)+g_{20} q(0) e^{i \omega_{0} \theta}+\bar{g}_{02} \bar{q}(0) e^{-i \omega_{0} \theta} .
\end{aligned}
$$

Solving for $W_{20}(\theta)$, we get

$$
W_{20}(\theta)=\frac{i g_{20} q(0)}{\omega_{0}} e^{i \omega_{0} \theta}+\frac{i \bar{g}_{02} \bar{q}(0)}{3 \omega_{0}} e^{-i \omega_{0} \theta}+\widehat{E}_{1} e^{2 i \omega_{0} \theta}
$$

And similarly from (69), (101), and (104), it follows that

$$
W_{11}(\theta)=\frac{-i g_{11} q(0)}{\omega_{0}} e^{i \omega_{0} \theta}+\frac{i \bar{g}_{11} \bar{q}(0)}{\omega_{0}} e^{-i \omega_{0} \theta}+\widehat{E}_{2},
$$

where $\widehat{E}_{1}=\left(E_{1}^{(1)}, E_{1}^{(2)}, E_{1}^{(3)}\right)^{T}$ and $\widehat{E}_{2}=\left(E_{2}^{(1)}, E_{2}^{(2)}, E_{2}^{(3)}\right)^{T}$ are both three dimensional constant vectors and can be determined by setting $\theta=0$ in $H(z, \bar{z}, \theta)$. This leads to

$$
\begin{aligned}
& H(z, \bar{z}, 0) \\
& =-2 \operatorname{Re}\left(\bar{q}^{*}(0) f_{0}(z, \bar{z}) q(0)\right)+f_{0}(z, \bar{z}) \\
& =-2 \operatorname{Re}(g(z, \bar{z}) q(0))+f_{0}(z, \bar{z}) \\
& =-\mathrm{g}(z, \bar{z}) q(0)-\bar{g}(z, \bar{z}) \bar{q}(0)+f_{0}(z, \bar{z}) \\
& =-\left(g_{20} \frac{z^{2}}{2}+g_{11} z \bar{z}+g_{02} \frac{\bar{z}^{2}}{2}+g_{21} \frac{z^{2} \bar{z}}{2}+\cdots\right) q(0) \\
& -\left(\bar{g}_{20} \frac{\bar{z}^{2}}{2}+\bar{g}_{11} z \bar{z}+g_{02} \frac{z^{2}}{2}+\bar{g}_{21} \frac{\bar{z}^{2} z}{2}+\cdots\right) \bar{q}(0) \\
& +\left[\begin{array}{l}
\Delta_{11} z^{2}+\Delta_{12} z \bar{z}+\Delta_{13} \bar{z}^{2}+\Delta_{14} z^{2} \bar{z} \\
\Delta_{21} z^{2}+\Delta_{22} z \bar{z}+\Delta_{23} \bar{z}^{2}+\Delta_{24} z^{2} \bar{z} \\
\Delta_{31} z^{2}+\Delta_{32} z \bar{z}+\Delta_{33} \bar{z}^{2}+\Delta_{34} z^{2} \bar{z}
\end{array}\right]+\cdots .
\end{aligned}
$$

Comparing the corresponding coefficients of the above equation with (99) for $\theta=0$, we get

$$
\begin{aligned}
& H_{20}(0)=-g_{20} q(0)-\bar{g}_{02} \bar{q}(0)+2\left(\begin{array}{c}
\Delta_{11} \\
\Delta_{21} \\
\Delta_{31}
\end{array}\right), \\
& H_{11}(0)=-g_{11} q(0)-\bar{g}_{11} \bar{q}(0)+2\left(\begin{array}{c}
\Delta_{12} \\
\Delta_{22} \\
\Delta_{32}
\end{array}\right) .
\end{aligned}
$$

From the definition of $A$ and (101) for $\theta=0$, we get

$$
\begin{gathered}
\int_{-\tau_{*}}^{0} d \eta(\theta) W_{20}(\theta)=2 i \omega_{0} W_{20}(0)-H_{20}(0), \\
\int_{-\tau_{*}}^{0} d \eta(\theta) W_{11}(\theta)=-H_{11}(0) .
\end{gathered}
$$

Notice that

$$
\begin{gathered}
\left(i \omega_{0} I-\int_{-\tau_{*}}^{0} e^{i \omega_{0} \theta} d \eta(\theta)\right) q(0)=0 \\
\left(-i \omega_{0} I-\int_{-\tau_{*}}^{0} e^{-i \omega_{0} \theta} d \eta(\theta)\right) \bar{q}(0)=0 .
\end{gathered}
$$

Substituting (106) and (111) into (109), we obtain

$$
\left(2 i \omega_{0} I-\int_{-\tau_{*}}^{0} e^{2 i \omega_{0} \theta} d \eta(\theta)\right) \widehat{E}_{1}=2\left(\begin{array}{c}
\Delta_{11} \\
\Delta_{21} \\
\Delta_{31}
\end{array}\right)
$$

which leads to

$$
\left(2 i \omega_{0} I-B_{1}-B_{2} e^{-2 i \omega_{0} \tau_{*}}\right) \widehat{E}_{1}=2\left(\begin{array}{c}
\Delta_{11} \\
\Delta_{21} \\
\Delta_{31}
\end{array}\right)
$$

that is,

$$
\begin{aligned}
& \left(\begin{array}{ccc}
2 i \omega_{0}-a_{11} & -a_{12} & 0 \\
-a_{21} & 2 i \omega_{0}-a_{22} & b_{23} e^{-2 i \omega_{0} \tau_{*}} \\
-a_{31} & -a_{32} & 2 i \omega_{0}-a_{33}
\end{array}\right)\left(\begin{array}{c}
E_{1}^{(1)} \\
E_{1}^{(2)} \\
E_{1}^{(3)}
\end{array}\right) \\
& \quad=2\left(\begin{array}{c}
\Delta_{11} \\
\Delta_{21} \\
\Delta_{31}
\end{array}\right) .
\end{aligned}
$$

On simplification, we get

$$
\begin{gathered}
E_{1}^{(1)}=\frac{B_{11} C_{22}-B_{22} C_{11}}{A_{11} B_{22}-A_{22} B_{11}}, \\
E_{1}^{(2)}=\frac{\left(2 i \omega_{0}-a_{11}\right) E_{1}^{(1)}-2 \Delta_{11}}{a_{12}}, \\
E_{1}^{(3)}=\frac{C_{11} A_{22}-A_{11} C_{22}}{A_{11} B_{22}-A_{22} B_{11}},
\end{gathered}
$$


where

$$
\begin{gathered}
A_{11}=-a_{21}+\frac{\left(2 i \omega_{0}-a_{22}\right)\left(2 i \omega_{0}-a_{11}\right)}{a_{12}}, \\
B_{11}=-b_{23} e^{-2 i \omega_{0} \tau_{*}}, \\
C_{11}=-\frac{2 \Delta_{11}}{a_{12}}\left(2 i \omega_{0}-a_{22}\right), \\
A_{22}=-a_{31}-\frac{a_{32}}{a_{12}}\left(2 i \omega_{0}-a_{11}\right), \\
B_{22}=2 i \omega_{0}-a_{33}, \\
C_{22}=\frac{2 \Delta_{11} a_{32}}{a_{12}}-2 \Delta_{31} .
\end{gathered}
$$

Similarly substituting (107) and (112) into (110), we get

$$
\left(-\int_{-\tau_{*}}^{0} d \eta(\theta)\right) \widehat{E}_{2}=2\left(\begin{array}{c}
\Delta_{12} \\
\Delta_{22} \\
\Delta_{32}
\end{array}\right)
$$

which leads to

$$
\left(-B_{1}-B_{2}\right) \widehat{E}_{2}=2\left(\begin{array}{l}
\Delta_{12} \\
\Delta_{22} \\
\Delta_{32}
\end{array}\right)
$$

that is,

$$
\begin{gathered}
\left(\begin{array}{ccc}
-a_{11} & -a_{12} & 0 \\
-a_{21} & -a_{22} & -b_{23} \\
a_{31} & -a_{32} & -a_{33}
\end{array}\right)\left(\begin{array}{c}
E_{2}^{(1)} \\
E_{2}^{(2)} \\
E_{2}^{(3)}
\end{array}\right) \\
=2\left(\begin{array}{c}
\Delta_{12} \\
\Delta_{22} \\
\Delta_{32}
\end{array}\right) .
\end{gathered}
$$

On simplification, we get

$$
\begin{gathered}
E_{2}^{(1)}=-\frac{B_{11}^{\prime} C_{22}^{\prime}-B_{22}^{\prime} C_{11}^{\prime}}{A_{11}^{\prime} B_{22}^{\prime}-A_{22}^{\prime} B_{11}^{\prime}}, \\
E_{2}^{(2)}=\frac{a_{11} E_{2}^{(1)}-2 \Delta_{12}}{a_{12}}, \\
E_{2}^{(3)}=\frac{C_{11}^{\prime} A_{22}^{\prime}-C_{22}^{\prime} A_{11}^{\prime}}{A_{11}^{\prime} B_{22}^{\prime}-A_{22}^{\prime} B_{11}^{\prime}},
\end{gathered}
$$

where

$$
\begin{gathered}
A_{11}^{\prime}=-a_{21}+\frac{a_{11} a_{22}}{a_{12}}, \\
B_{11}^{\prime}=-b_{23}, \\
C_{11}^{\prime}=2\left(\frac{\Delta_{12} a_{22}}{a_{12}}-\Delta_{22}\right), \\
A_{22}^{\prime}=-a_{31}+\frac{a_{11} a_{32}}{a_{12}}, \\
B_{22}^{\prime}=-a_{33}, \\
C_{22}^{\prime}=2\left(\frac{\Delta_{12} a_{32}}{a_{12}}-\Delta_{32}\right) .
\end{gathered}
$$

Thus, $W_{20}(\theta)$ and $W_{11}(\theta)$ can be determined from (90) and (93). Furthermore, $g_{21}$ can also be determined. Therefore, based on the previous analysis, $g_{i j}$ can be determined in terms of the parameters and delays involved in the system. Thus, we can compute the following values:

$$
C_{1}(0)=\frac{i}{2 \omega_{0}}\left(g_{11} g_{20}-2\left|g_{11}\right|^{2}-\frac{\left|g_{02}\right|^{2}}{3}\right)+\frac{g_{21}}{2}
$$

$$
\begin{gathered}
\mu_{2}=-\frac{\operatorname{Re}\left\{C_{1}(0)\right\}}{\operatorname{Re}\left\{\lambda^{\prime}\left(\tau_{0}\right)\right\}}, \\
\beta_{2}=2 \operatorname{Re}\left\{C_{1}(0)\right\}, \\
T_{2}=-\frac{\operatorname{Im}\left\{C_{1}(0)\right\}+\mu_{2} \operatorname{Im}\left\{\lambda^{\prime}\left(\tau_{0}\right)\right\}}{\omega_{0}},
\end{gathered}
$$

where sign of $\mu_{2}$ determines the direction of the Hopf bifurcation, sign of $\beta_{2}$ determines the stability of the bifurcating periodic solution, and sign of $T_{2}$ determines the period of the bifurcating periodic solution in the center manifold at the critical value $\tau_{*}$. Therefore, by the results of Hassard et al. [33], we can summarize the properties of the Hopf bifurcation at the critical value $\tau=\tau_{*}$ in the following theorem.

Theorem 8. In system (31) and (32), the following results hold.

(i) If $\mu_{2}>0(<0)$, the Hopf bifurcation is supercritical (subcritical).

(ii) If $\beta_{2}<0(>0)$, the bifurcated periodic solutions are stable (unstable).

(iii) If $T_{2}>0(<0)$, period of the bifurcating periodic solution increases (decreases).

\section{Model with Double Delays}

In this section we have discussed the dynamical behaviours of the model (3) and (4) with double discrete time delays $\tau_{1}$ and $\tau_{2}$. Here, $\tau_{1}$ represents the activation period or reaction time of the toxicant in the population biomass; $\tau_{2}$ represents the activation period or reaction time of the toxicant in the 
population from the environment and $\gamma(t)=Q(>0)$ is a constant. The modified model is as follows:

$$
\begin{gathered}
\frac{d X}{d t}=X\left(p-Y\left(t-\tau_{1}\right)-X\right) \\
\frac{d Y}{d t}=Z\left(t-\tau_{2}\right)-(q-X) Y \\
\frac{d Z}{d t}=-a X Z+a(d+Y) X Y-h Z+Q
\end{gathered}
$$

with initial conditions

$$
\begin{gathered}
X(0)>0, \quad Y(\vartheta)=\varphi_{1}(\vartheta), \\
Z(\vartheta)=\varphi_{2}(\vartheta), \\
\text { such that } \varphi_{1}(\vartheta) \geq 0, \quad \forall \vartheta \in\left[-\tau_{1}, 0\right], \\
\varphi_{2}(\vartheta) \geq 0, \quad \forall \vartheta \in\left[-\tau_{2}, 0\right],
\end{gathered}
$$

where $\varphi_{1}(\vartheta)$ is a continuous function on $\vartheta \in\left[-\tau_{1}, 0\right]$ and $\varphi_{2}(\vartheta)$ is a continuous function on $\vartheta \in\left[-\tau_{2}, 0\right]$. For a biological meaning, we further assume that $\varphi_{i}(0)>0(i=1,2)$.

Now, we will estimate the length of the delay which preserves the stability of the system (125a) and (125b). The corresponding linearized system of (125a) and (125b) about the interior equilibrium $E^{*}\left(X^{*}, Y^{*}, Z^{*}\right)$ is given by

$$
\begin{gathered}
\frac{d x_{1}}{d t}=a_{11} x_{1}+a_{12} y_{1}\left(t-\tau_{1}\right) \\
\frac{d y_{1}}{d t}=a_{21} x_{1}+a_{22} y_{1}+b_{23} z_{1}\left(t-\tau_{2}\right) \\
\frac{d z_{1}}{d t}=a_{31} x_{1}+a_{32} y_{1}+a_{33} z_{1},
\end{gathered}
$$

where

$$
\begin{gathered}
X(t)=x_{1}(t)+X^{*}, \quad Y(t)=y_{1}(t)+Y^{*}, \\
Z(t)=z_{1}(t)+Z^{*} .
\end{gathered}
$$

Taking Laplace transform of the system (126), we get

$$
\begin{aligned}
& \left(s-a_{11}\right) \overline{x_{1}}(s)=a_{12} e^{-s \tau_{1}} \overline{y_{1}}(s)+a_{12} e^{-s \tau_{1}} k_{1}(s)+x_{1}(0) \\
& \left(s-a_{22}\right) \overline{y_{1}}(s) \\
& \quad=a_{21} \overline{x_{1}}(s)+b_{23} e^{-s \tau_{2}} \overline{z_{1}}(s)+b_{23} e^{-s \tau_{2}} k_{1}(s)+y_{1}(0) \\
& \quad\left(s-a_{33}\right) \overline{z_{1}}(s)=a_{31} \overline{x_{1}}(s)+a_{32} \overline{y_{1}}(s)+z_{1}(0),
\end{aligned}
$$

where

$$
\begin{aligned}
& k_{1}(s)=\int_{-\tau_{1}}^{0} e^{-s t} y_{1}(t) d t, \\
& k_{2}(s)=\int_{-\tau_{2}}^{0} e^{-s t} z_{1}(t) d t,
\end{aligned}
$$

and $\overline{x_{1}}(s), \overline{y_{1}}(s), \overline{z_{1}}(s)$ are the Laplace transforms of $x_{1}(t)$, $y_{1}(t)$, and $z_{1}(t)$, respectively.
Now, using "Nyquist theorem" [21, 31], it can be shown that the conditions for local asymptotic stability of $E^{*}\left(X^{*}, Y^{*}\right.$, and $Z^{*}$ ) are given by [32]

$$
\begin{aligned}
& \operatorname{Im} H\left(i \eta_{0}\right)>0, \\
& \operatorname{Re} H\left(i \eta_{0}\right)=0,
\end{aligned}
$$

where

$$
\begin{gathered}
H(s)=s^{3}+D_{1} s^{2}+D_{2} s+D_{3}+D_{4} s e^{-s \tau_{1}}+D_{5} s e^{-s \tau_{2}} \\
+D_{6} e^{-s \tau_{1}}+D_{7} e^{-s \tau_{2}}+D_{8} e^{-s\left(\tau_{1}+\tau_{2}\right)}, \\
D_{1}=a X^{*}+h+q, \\
D_{2}=a q X^{*}+h q+q X^{*}-\left(X^{*}\right)^{2}, \\
D_{3}=a q\left(X^{*}\right)^{2}+q h X^{*}-a\left(X^{*}\right)^{3}-h\left(X^{*}\right)^{2}, \\
D_{4}=X^{*} Y^{*}, \\
D_{5}=-a\left(d+Y^{*}\right) X^{*}-a X^{*} Y^{*}, \\
D_{6}=X^{*} Y^{*}\left(a X^{*}+h\right), \\
D_{7}=-X^{*}\left[a\left(d+Y^{*}\right) X^{*}-a X^{*} Y^{*}\right], \\
D_{8}=X^{*}\left[-a Z^{*}+a\left(d+Y^{*}\right) Y^{*}\right]
\end{gathered}
$$

and $\eta_{0}$ is the smallest positive root of (131).

Nyquist criterion $[21,31]$ implies that if $\eta_{0}$ is a solution of (131), then

$$
\begin{gathered}
-D_{1} \eta_{0}^{2}+D_{3}+D_{4} \eta_{0} \sin \left(\eta_{0} \tau_{1}\right)+D_{5} \eta_{0} \sin \left(\eta_{0} \tau_{2}\right) \\
+D_{6} \cos \left(\eta_{0} \tau_{1}\right)+D_{7} \cos \left(\eta_{0} \tau_{2}\right) \\
+D_{8} \cos \left\{\eta_{0}\left(\tau_{1}+\tau_{2}\right)\right\}=0 .
\end{gathered}
$$

Next, we wish to find an upper bound for $\eta$ (say, $\eta_{+}$) independent of $\tau_{1}, \tau_{2}$ such that (130) and (131) hold for all $\eta, 0 \leq \eta \leq \eta_{+}$ and hence in particular for $\eta_{0}$ when $\eta_{0} \leq \eta_{+}$.

Maximizing (133) with $\left|\sin \left(\eta_{0} \tau_{1}\right)\right| \leq 1,\left|\sin \left(\eta_{0} \tau_{2}\right)\right| \leq 1$, $\left|\cos \left(\eta_{0} \tau_{1}\right)\right| \leq 1,\left|\cos \left(\eta_{0} \tau_{2}\right)\right| \leq 1,\left|\cos \left\{\eta_{0}\left(\tau_{1}+\tau_{2}\right)\right\}\right| \leq 1$, we get

$$
D_{1} \eta_{0}^{2}-\left(\left|D_{4}\right|+\left|D_{5}\right|\right) \eta_{0}-\left(\left|D_{3}\right|+\left|D_{6}\right|+\left|D_{7}\right|+\left|D_{8}\right|\right)=0 \text {. }
$$

If $\eta_{+}$is a positive root of (134), then we obtain

$$
\begin{aligned}
\eta_{+}=\frac{1}{2 D_{1}}[ & \left(\left|D_{4}\right|+\left|D_{5}\right|\right) \\
+ & \left\{\left(\left|D_{4}\right|+\left|D_{5}\right|\right)^{2}\right. \\
& \left.\left.+4 D_{1}\left(\left|D_{3}\right|+\left|D_{6}\right|+\left|D_{7}\right|+\left|D_{8}\right|\right)\right\}^{1 / 2}\right] .
\end{aligned}
$$

Then, clearly, $\eta_{+} \geq \eta_{0}$.

Equation (130) indicates that the following inequality should hold:

$$
\varphi\left(\tau_{1}, \tau_{2}, \eta_{0}\right)>\psi\left(\tau_{1}, \tau_{2}, \eta_{0}\right)
$$


where

$$
\begin{aligned}
& \varphi\left(\tau_{1}, \tau_{2}, \eta_{0}\right) \\
& =-\eta_{0}\left[\eta_{0}^{2}-D_{2}-D_{4} \cos \left(\eta_{0} \tau_{1}\right)-D_{5} \cos \left(\eta_{0} \tau_{2}\right)\right] \\
& \psi\left(\tau_{1}, \tau_{2}, \eta_{0}\right) \\
& =D_{6} \sin \left(\eta_{0} \tau_{1}\right)+D_{7} \sin \left(\eta_{0} \tau_{2}\right) \\
& \quad+D_{8} \sin \left\{\eta_{0}\left(\tau_{1}+\tau_{2}\right)\right\} .
\end{aligned}
$$

If we can find $\widetilde{\varphi}\left(\tau_{1}, \tau_{2}\right)>\widetilde{\psi}\left(\tau_{1}, \tau_{2}\right)$ such that

$$
\frac{\varphi\left(\tau_{1}, \tau_{2}, \eta_{0}\right)}{\left(\tau_{1}+\tau_{2}\right) \eta_{0}} \geq \tilde{\varphi}\left(\tau_{1}, \tau_{2}\right)>\widetilde{\psi}\left(\tau_{1}, \tau_{2}\right) \geq \frac{\psi\left(\tau_{1}, \tau_{2}, \eta_{0}\right)}{\left(\tau_{1}+\tau_{2}\right) \eta_{0}},
$$

where $0<\eta_{0}<\eta_{+}$, then the Nyquist criterion holds. By (138), we can estimate the values of $\tau_{1}$ and $\tau_{2}$

$$
\begin{aligned}
& \frac{\psi\left(\tau_{1}, \tau_{2}, \eta_{0}\right)}{\left(\tau_{1}+\tau_{2}\right) \eta_{0}} \\
& \quad=\frac{D_{6} \sin \left(\eta_{0} \tau_{1}\right)+D_{7} \sin \left(\eta_{0} \tau_{2}\right)+D_{8} \sin \left\{\eta_{0}\left(\tau_{1}+\tau_{2}\right)\right\}}{\left(\tau_{1}+\tau_{2}\right) \eta_{0}} \\
& \quad \leq\left|D_{6}\right|+\left|D_{7}\right|+\left|D_{8}\right| .
\end{aligned}
$$

We choose

$$
\widetilde{\psi}\left(\tau_{1}, \tau_{2}\right)=\left|D_{6}\right|+\left|D_{7}\right|+\left|D_{8}\right|
$$

Now,

$$
\begin{aligned}
\frac{\varphi\left(\tau_{1}, \tau_{2}, \eta_{0}\right)}{\left(\tau_{1}+\tau_{2}\right) \eta_{0}} & =\frac{\eta_{0}\left\{D_{2}+D_{4} \cos \left(\eta_{0} \tau_{1}\right)+D_{5} \cos \left(\eta_{0} \tau_{2}\right)-\eta_{0}^{2}\right\}}{\left(\tau_{1}+\tau_{2}\right) \eta_{0}} \\
& \geq \frac{\left\{D_{2}+D_{4} \cos \left(\eta_{+} \tau_{1}\right)+\left|D_{5}\right|-\eta_{+}^{2}\right\}}{\left(\tau_{1}+\tau_{2}\right)} .
\end{aligned}
$$

We can choose

$$
\widetilde{\varphi}\left(\tau_{1}, \tau_{2}\right)=\frac{\left\{D_{2}+D_{4} \cos \left(\eta_{+} \tau_{1}\right)+\left|D_{5}\right|-\eta_{+}^{2}\right\}}{\left(\tau_{1}+\tau_{2}\right)} .
$$

From (138), we get

$$
\begin{aligned}
\tilde{\varphi}\left(\tau_{1}, \tau_{2}\right) & =\frac{\left\{D_{2}+D_{4} \cos \left(\eta_{+} \tau_{1}\right)+\left|D_{5}\right|-\eta_{+}^{2}\right\}}{\left(\tau_{1}+\tau_{2}\right)} \\
& >\left|D_{6}\right|+\left|D_{7}\right|+\left|D_{8}\right|=\widetilde{\psi}\left(\tau_{1}, \tau_{2}\right) .
\end{aligned}
$$

Thus

$$
\begin{aligned}
\tau_{1}+\tau_{2} & <\frac{D_{2}+D_{4} \cos \left(\eta_{+} \tau_{1}\right)+\left|D_{5}\right|-\eta_{+}^{2}}{\left|D_{6}\right|+\left|D_{7}\right|+\left|D_{8}\right|} \\
& \leq \frac{\left|D_{2}\right|+\left|D_{4}\right|+\left|D_{5}\right|-\eta_{+}^{2}}{\left|D_{6}\right|+\left|D_{7}\right|+\left|D_{8}\right|} .
\end{aligned}
$$

So, we come to the following theorem.
Theorem 9. The delayed model (125a) and (125b) will be locally asymptotically stable at $E^{*}\left(X^{*}, Y^{*}, Z^{*}\right)$ with the conditions (24) if

$$
\begin{gathered}
\left|D_{2}\right|+\left|D_{4}\right|+\left|D_{5}\right|>\eta_{+}^{2}, \\
0<\tau_{1}+\tau_{2}<\frac{\left|D_{2}\right|+\left|D_{4}\right|+\left|D_{5}\right|-\eta_{+}^{2}}{\left|D_{6}\right|+\left|D_{7}\right|+\left|D_{8}\right|} .
\end{gathered}
$$

Next, we will study the existence of Hopf bifurcation of system (125a) and (125b) by choosing one of the delays as a bifurcation parameter. Let us take $\tau_{2}$ as a bifurcation parameter. The characteristic equation corresponding to system (125a) and (125b) is

$$
\begin{aligned}
\lambda^{3}+ & D_{1} \lambda^{2}+D_{2} \lambda+D_{3}+D_{4} \lambda e^{-\lambda \tau_{1}}+D_{5} \lambda e^{-\lambda \tau_{2}}+D_{6} e^{-\lambda \tau_{1}} \\
& +D_{7} e^{-\lambda \tau_{2}}+D_{8} e^{-\lambda\left(\tau_{1}+\tau_{2}\right)}=0
\end{aligned}
$$

Hence, $\lambda= \pm i \eta$ is a simple root of (146) at $\tau_{2}=\tau_{2}^{c}$. By separating real and imaginary parts, we obtain

$$
\begin{aligned}
& -D_{1} \eta^{2}+D_{3}+D_{4} \eta \sin \left(\eta \tau_{1}\right)+D_{5} \eta \sin \left(\eta \tau_{2}\right) \\
& +D_{6} \cos \left(\eta \tau_{1}\right)+D_{7} \cos \left(\eta \tau_{2}\right)+D_{8} \cos \left\{\eta\left(\tau_{1}+\tau_{2}\right)\right\}=0, \\
& -\eta^{3}+D_{2} \eta+D_{4} \eta \cos \left(\eta \tau_{1}\right)+D_{5} \eta \cos \left(\eta \tau_{2}\right) \\
& \quad-D_{6} \sin \left(\eta \tau_{1}\right)-D_{7} \sin \left(\eta \tau_{2}\right)+D_{8} \sin \left\{\eta\left(\tau_{1}+\tau_{2}\right)\right\}=0 .
\end{aligned}
$$

Differentiating equation (146) implicitly with respect to $\tau_{2}$, we obtain

$$
\begin{aligned}
\left(\frac{d \lambda}{d \tau_{2}}\right)^{-1}= & \frac{D_{5}}{\lambda\left(D_{5} \lambda+D_{7}+D_{8} e^{\left.-\lambda \tau_{1}\right)}\right.} \\
& +\frac{D_{8} \tau_{1} e^{-\lambda \tau_{1}}}{\lambda\left(D_{5} \lambda+D_{7}+D_{8} e^{\left.-\lambda \tau_{1}\right)}\right.} \\
& +\frac{3 \lambda^{2}+2 D_{1} \lambda+D_{2}}{\lambda e^{-\lambda \tau_{2}}\left(D_{5} \lambda+D_{7}+D_{8} e^{\left.-\lambda \tau_{1}\right)}\right.} \\
& +\frac{e^{-\lambda \tau_{1}}\left(D_{4}-D_{4} \lambda \tau_{1}-D_{6} \tau_{1}\right)}{\lambda e^{-\lambda \tau_{2}}\left(D_{5} \lambda+D_{7}+D_{8} e^{-\lambda \tau_{1}}\right)}-\frac{\tau_{2}}{\lambda}
\end{aligned}
$$

Now,

$$
\operatorname{Re}\left(\frac{d \lambda}{d \tau_{2}}\right)_{\lambda=i \eta_{0}}^{-1}=\frac{1}{M^{2}+N^{2}}(M P+N R),
$$


where

$$
\begin{aligned}
& M=-D_{5} \eta_{0}^{2}+D_{8} \eta_{0} \sin \left(\eta_{0} \tau_{1}\right), \\
P=D_{7} \eta_{0}+D_{8} \eta_{0} \cos \left(\eta_{0} \tau_{1}\right), & D_{5}-D_{8} \tau_{1} \cos \left(\eta_{0} \tau_{1}\right)+\left(-3 \eta_{0}^{2}+D_{2}\right) \cos \left(\eta_{0} \tau_{2}\right) \\
- & 2 D_{1} \eta_{0} \sin \left(\eta_{0} \tau_{2}\right) \\
+ & \left\{\left(D_{4}-D_{6} \tau_{1}\right) \cos \left(\eta_{0} \tau_{1}\right)-\eta_{0} \tau_{1} D_{4} \sin \left(\eta_{0} \tau_{1}\right)\right\} \\
\times & \cos \left(\eta_{0} \tau_{2}\right) \\
+ & \left\{\left(D_{4}-D_{6} \tau_{1}\right) \sin \left(\eta_{0} \tau_{1}\right)+\eta_{0} \tau_{1} D_{4} \cos \left(\eta_{0} \tau_{1}\right)\right\} \\
\times & \sin \left(\eta_{0} \tau_{2}\right) \\
R= & D_{8} \tau_{1} \sin \left(\eta_{0} \tau_{1}\right)+\left(-3 \eta_{0}^{2}+D_{2}\right) \sin \left(\eta_{0} \tau_{2}\right) \\
+ & 2 D_{1} \eta_{0} \cos \left(\eta_{0} \tau_{2}\right) \\
+ & \left\{\left(D_{4}-D_{6} \tau_{1}\right) \cos \left(\eta_{0} \tau_{1}\right)-\eta_{0} \tau_{1} D_{4} \sin \left(\eta_{0} \tau_{1}\right)\right\} \\
\times & \sin \left(\eta_{0} \tau_{2}\right) \\
- & \left\{\left(D_{4}-D_{6} \tau_{1}\right) \sin \left(\eta_{0} \tau_{1}\right)+\eta_{0} \tau_{1} D_{4} \sin \left(\eta_{0} \tau_{1}\right)\right\} \\
\times & \times \cos \left(\eta_{0} \tau_{2}\right) .
\end{aligned}
$$

Cleary, the sign of $M^{2}+N^{2}$ in (149) is positive. Because of the coherence of the sign of $M P+N R$ and $\operatorname{Re}\left(d \lambda / d \tau_{2}\right)_{\lambda=i \eta_{0}}^{-1}$, we get that if $M P+N R>0$, then $\operatorname{Re}\left(d \lambda / d \tau_{2}\right)_{\lambda=i \eta_{0}}^{-1}>0$.

Therefore, transversality condition holds and Hopf bifurcation occurs at $\tau_{2}=\tau_{2}^{c}$, provided that $(M P+N R)>0$. result.

Summarizing the above analysis, we get the following

Theorem 10. If there exists $\tau_{2}=\tau_{2}^{c}$ such that (147) holds and $\operatorname{Re}\left(d \lambda / d \tau_{2}\right)_{\lambda=i \eta_{0}}^{-1}>0$, that is, $(M P+N R)>0$, then a Hopf bifurcation occurs at $E^{*}\left(X^{*}, Y^{*}, Z^{*}\right)$ as $\tau_{2}$ passes through $\tau_{2}^{c}$.

\section{Numerical Simulations}

Analytical studies can never be completed without numerical verification of the derived results. In this section, we present computer simulation of some important analytical results discussed in the previous sections. Besides verification of our analytical findings, these numerical solutions are very important from a practical point of view.

We take the parameters of the system as given in Table 1. Using those values under zero exogenous input, that is, $\gamma=$ 0 , it is observed that the equilibrium $E_{0}(0,0,0)$ becomes unstable and interior equilibrium $E^{*}\left(X^{*}, Y^{*}, Z^{*}\right)$ is not feasible. Only the axial equilibrium $E_{1}(p, 0,0)=(2.28,0,0)$ is locally asymptotically stable, which is shown in Figure 1. Therefore, Theorem 2 is verified. When $\gamma=1.25$ (i.e., nonzero exogenous input) using the parameter values given in Table 2, it is observed that the equilibrium $E_{2}=(0,4.28082,12.5)$ exists and is locally asymptotically stable with $h p q<\gamma$, but the interior equilibrium $E^{*}$ does not exist, which is shown
TABLE 1

\begin{tabular}{lc}
\hline Parameter & Values \\
\hline$p$ & 2.28 \\
$q$ & 2.92 \\
$a$ & 4.14 \\
$d$ & 0.54 \\
$h$ & 0.2 \\
\hline
\end{tabular}

TABLE 2

\begin{tabular}{lc}
\hline Parameter & Values \\
\hline$p$ & 2.28 \\
$q$ & 2.92 \\
$a$ & 4.14 \\
$d$ & 0.54 \\
$h$ & 0.1 \\
\hline
\end{tabular}

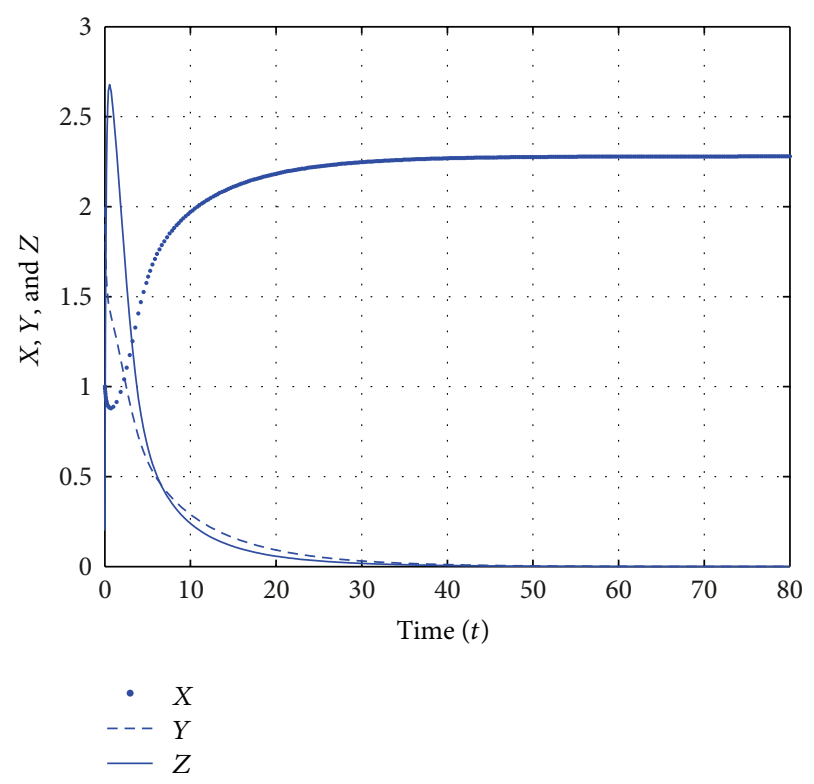

Figure 1: Time series plot of $X, Y$, and $Z$ for zero exogenous input $(\gamma=0)$ with $X(0)=1, Y(0)=2$, and $Z(0)=0.2$; parameter values are given in Table 1.

in Figure 2. On the other hand, when $\gamma=1.25$ using the parameter values given in Table 1 , it is found that $h p q>\gamma$, $a(q-p-d)>h$, and $E_{2}=(0,2.14041,6.25)$ becomes unstable and $E^{*}\left(X^{*}, Y^{*}, Z^{*}\right)=(0.43227,1.84773,4.59665)$ exists, which is shown in Figure 3. Therefore, Theorem 3 is verified.

Using the parameter values given in Table 1 with $\gamma=1.25$, it is observed that the conditions of Theorem 4 are satisfied as $A_{1}=4.9096>0, A_{3}=0.121507>0, A_{1} A_{2}-A_{3}=$ $0.388821>0$. Therefore, by Theorem $4, E^{*}$ is locally asymptotically stable. Figure 3 shows that $X, Y$ and $Z$ approach to their steady-state values $X^{*}, Y^{*}$, and $Z^{*}$, respectively, in finite time. The $X Y$-plane, $Y Z$-plane, $X Z$-plane, and $X Y Z$ plane projections of the solution are shown in Figures 4, 5, 6 , and 7, respectively. Here the conditions of Theorem 5 are 


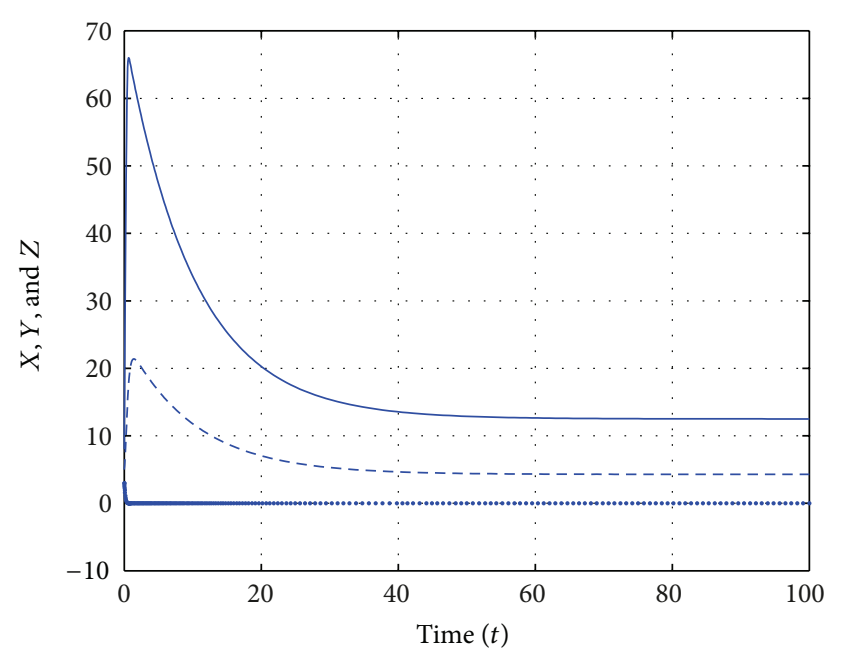

$-\quad X$
$---\quad Y$
$-\quad Z$

Figure 2: Time series plot of $X, Y$, and $Z$ for nonzero exogenous input $(\gamma=1.25)$ and $h p q<\gamma$ with $X(0)=3, Y(0)=5$, and $Z(0)=8$; parameter values are given in Table 2 .

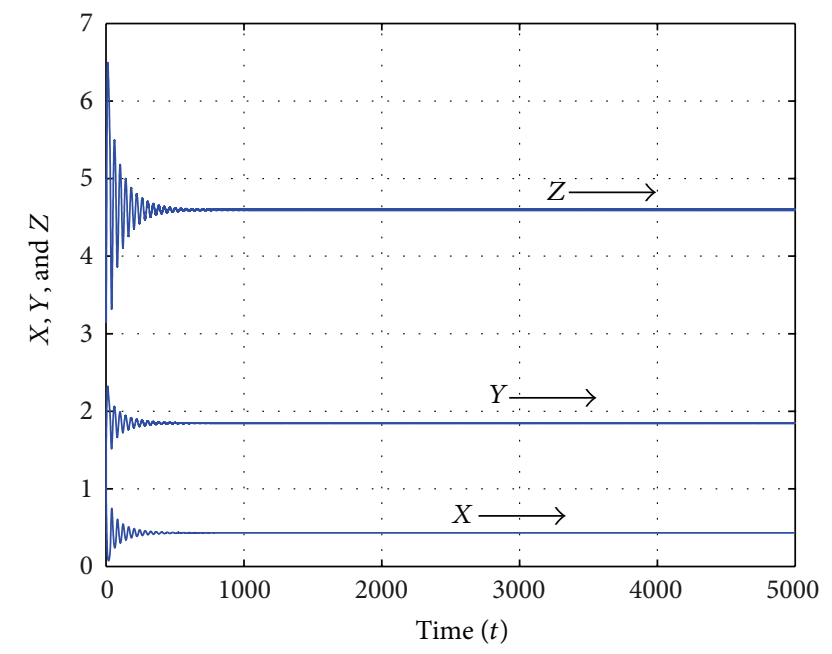

Figure 3: Time series plot of $X, Y$, and $Z$ for nonzero exogenous input $(\gamma=1.25)$ and $h p q>\gamma$ with $X(0)=1, Y(0)=1$, and $Z(0)=5$; parameter values are given in Table 1.

also satisfied and consequently $E^{*}$ is globally asymptotically stable. The phase diagram is shown in Figure 8.

\section{Discussions and Conclusions}

In this paper, we have developed a single-species population model under the influence of toxicant in the population and in the environment by means of ordinary differential equations in terms of their concentrations. It is shown that the model system (3) with (4) is bounded and the solutions are positive, which in turn implies that the system is ecologically well behaved. The analysis of the existence of various

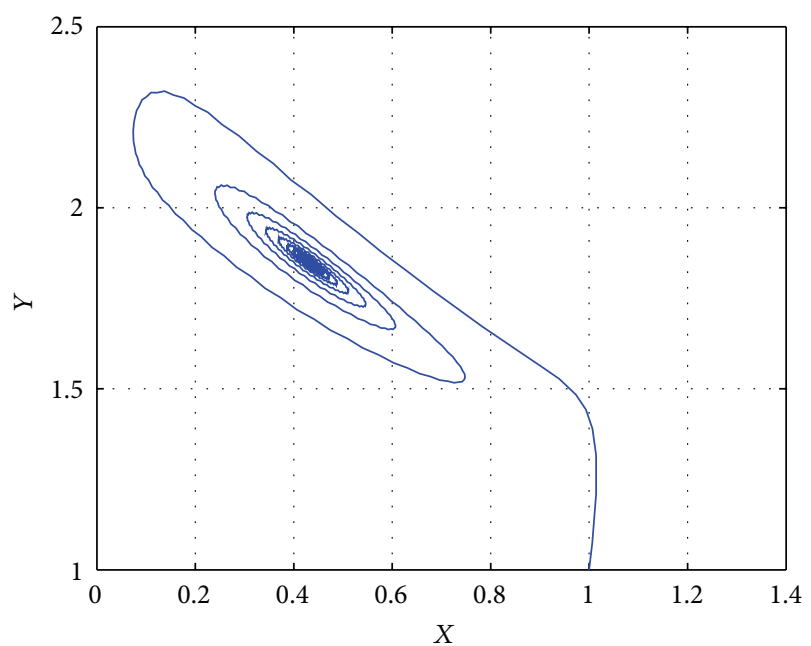

FIGURE 4: $X Y$-plane projection of the solution for nonzero exogenous input $(\gamma=1.25)$ with $X(0)=1, Y(0)=1$, and $Z(0)=5$; parameter values are given in Table 1.

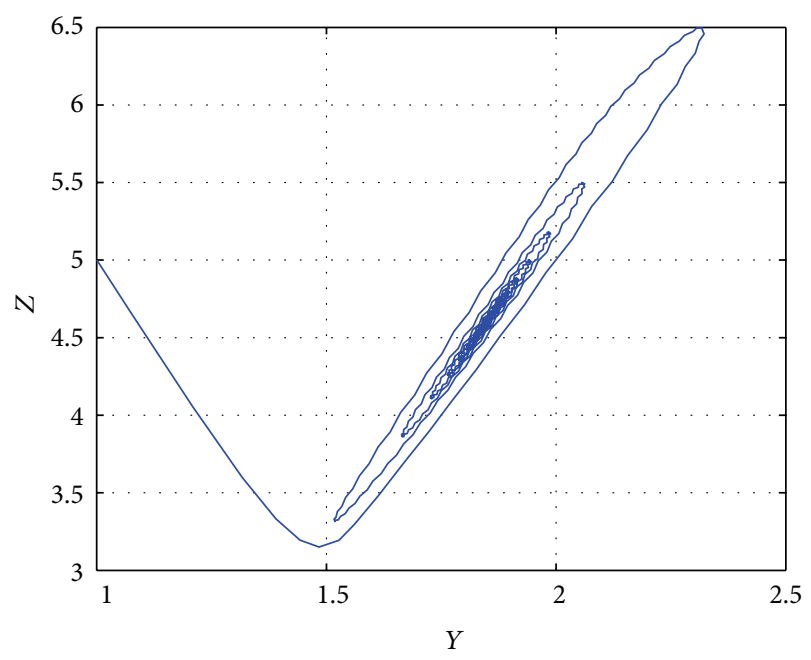

FIgURE 5: $Y Z$-plane projection of the solution for nonzero exogenous input $(\gamma=1.25)$ with $X(0)=1, Y(0)=1$, and $Z(0)=5$; parameter values are given in Table 1.

equilibrium points under zero exogenous toxicant input and nonzero exogenous toxicant input leads us to Theorems 2 and 3, respectively. Theorem 2 states that under zero exogenous input the trivial equilibrium $E_{0}$ is unstable, the interior equilibrium $E^{*}$ is not feasible, and only the axial equilibrium $E_{1}$ is locally asymptotically stable. On the other hand, when exogenous input is nonzero, the existence of the interior equilibrium $E^{*}$ depends on some conditions and the equilibrium $E_{2}$ is locally asymptotically stable under some condition. The stability criteria for $E^{*}$ given in Theorems 4 and 5 are the conditions for locally and globally stable coexistence of the population biomass, population toxicant, and environmental toxicant.

It is proved by several researchers that the effect of time delay must be taken into account in the population models to 


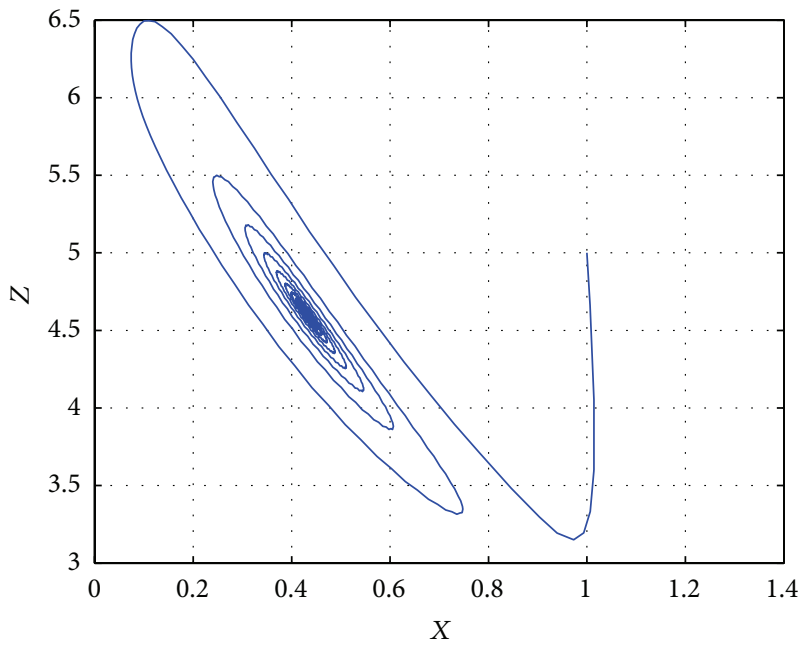

Figure 6: $X Z$-plane projection of the solution for nonzero exogenous input $(\gamma=1.25)$ with $X(0)=1, Y(0)=1$, and $Z(0)=5$; parameter values are given in Table 1.

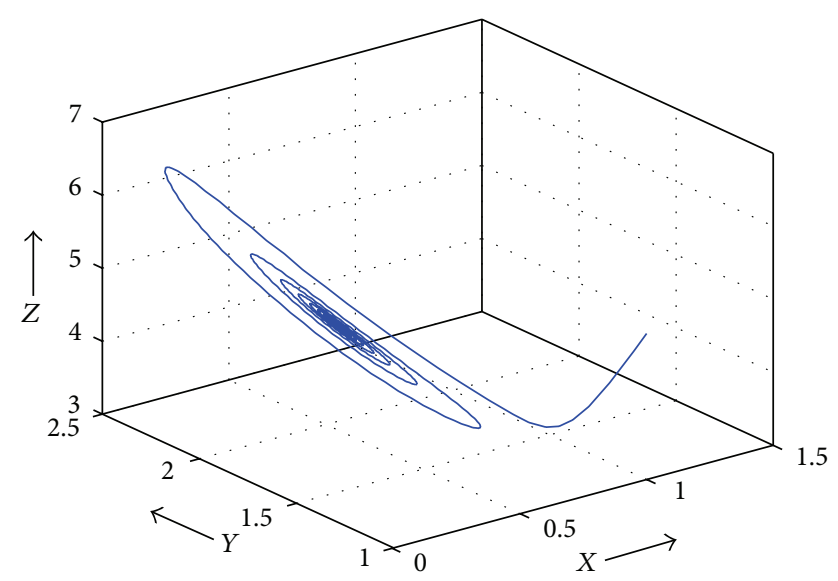

FIGURE 7: $X Y Z$-plane projection of the solution for nonzero exogenous input $(\gamma=1.25)$ with $X(0)=1, Y(0)=1$, and $Z(0)=5$; parameter values are given in Table 1.

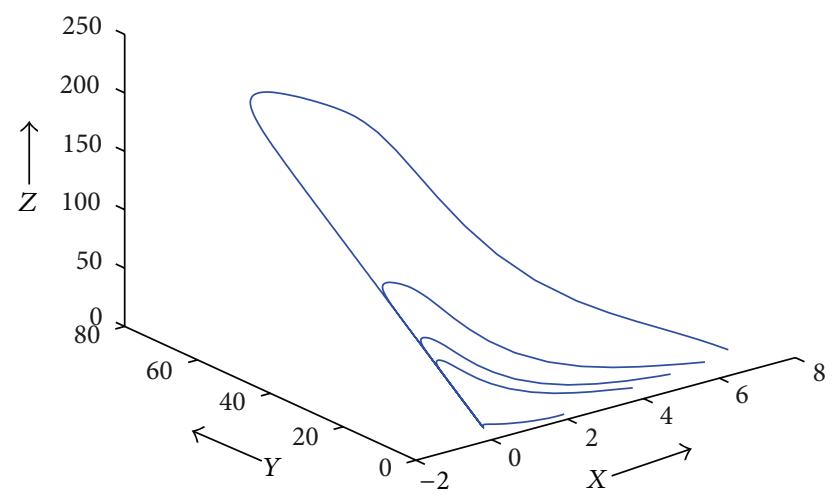

FIGURE 8: Global stability of $E^{*}\left(X^{*}, Y^{*}, Z^{*}\right)$ for nonzero exogenous input $(\gamma=1.25)$ with $(X(0), Y(0), Z(0))=(2,1,3),(4,3,5),(6,4,8)$, $(5,3,8),(7,8,4)$, parameter values are given in Table 1 . make them ecologically more meaningful and useful. It seems also reasonable that the effect of the environmental toxicant on the organismal activities and the population growth will not be instantaneous. It must be mediated with some discrete time lag required for incubation. From this point of view, we have formulated single and double time delayed model given in (31) and (125a) and (125b), respectively, where the delays may be looked upon as the reaction time of the environment toxicant on the population biomass and the population toxicant.

The analysis of the delayed models (31) and (125a) and (125b) shows more complicated behaviour than their nondelayed counterpart. The rigorous analysis of the single delayed model (31) leads us to Theorem 6 which mentions that the stability criteria in absence of delay are no longer enough to guarantee the stability in the presence of delay, but rather there is a critical value $\tau_{*}$ of the gestation delay $\tau$ such that the system is stable for $\tau<\tau_{*}$ and becomes unstable for $\tau>\tau_{*}$ at the interior equilibrium $E^{*}\left(X^{*}, Y^{*}, Z^{*}\right)$. From the next part of our analysis, we obtain the estimated length of delay to preserve stability at $E^{*}$ and the direction of Hopf bifurcation by using normal form theory and center manifold theorem. The stability of the double delayed model (125a) and (125b) is investigated by the Nyquist criteria which leads us to Theorem 9. By choosing one of the delays as a bifurcation parameter, the model (125a) and (125b) is found to undergo a Hopf bifurcation under some conditions stated in Theorem 10.

Analytical studies can never be completed without numerical verification of the results. So, some numerical simulations for justifying the important theoretical results are also illustrated by using MATLAB, which shows the mathematical and ecological reliability of the proposed model.

\section{References}

[1] T. G. Hallam, C. E. Clark, and G. S. Jordan, "The community matrix in three-species community models," Journal of Mathematical Biology, vol. 16, no. 1, pp. 25-31, 1983.

[2] T. G. Hallam, C. E. Clark, and R. R. Lassiter, "Effects of toxicants on populations: a qualitative approach. 1. Equilibrium environmental exposure," Ecological Modelling, vol. 18, no. 4, pp. 291304, 1983.

[3] T. G. Hallam and J. T. De Luna, "Effects of toxicants on populations: a qualitative approach. III. Environmental and food chain pathways," Journal of Theoretical Biology, vol. 109, no. 3, pp. 411429, 1984.

[4] Z. Ma, The Study of Biology Model, Anhui Education Press, He fei, China, 1996.

[5] M. Zhien, S. Baojun, and T. G. Hallam, "The threshold of survival for systems in a fluctuating environment," Bulletin of Mathematical Biology, vol. 51, no. 3, pp. 311-323, 1989.

[6] Z. He and Z. Ma, "On the effects of polution and catch to a logistic population," Journal of Biomathematics, vol. 14, pp. 281$287,1999$.

[7] Z. He and Z. Ma, "The effect of toxicant and harvest on the logistic model," Journal of Biomathematics, vol. 12, pp. 230-237, 1997.

[8] B. Buonomo, A. Di Liddo, and I. Sgura, "A diffusive-convective model for the dynamics of population-toxicant interactions: 
some analytical and numerical results," Mathematical Biosciences, vol. 157, no. 1-2, pp. 37-64, 1999.

[9] G. P. Samanta and A. Maiti, "Dynamical model of a singlespecies system in a polluted environment," Journal of Applied Mathematics \& Computing, vol. 16, no. 1-2, pp. 231-242, 2004.

[10] J. T. De Luna and T. G. Hallam, "Effects of toxicants on populations: a qualitative approach IV. Resource-consumer-toxicant models," Ecological Modelling, vol. 35, no. 3-4, pp. 249-273, 1987.

[11] H. I. Freedman and J. B. Shukla, "Models for the effect of toxicant in single-species and predator-prey systems," Journal of Mathematical Biology, vol. 30, no. 1, pp. 15-30, 1991.

[12] M. Ghosh, P. Chandra, and P. Sinha, "A mathematical model to study the effect of toxic chemicals on a prey-predator type fishery," Journal of Biological Systems, vol. 10, no. 2, pp. 97-105, 2002.

[13] J. He and K. Wang, "The survival analysis for a population in a polluted environment," Nonlinear Analysis. Real World Applications. An International Multidisciplinary Journal, vol. 10, no. 3, pp. 1555-1571, 2009.

[14] Z. Li, Z. Shuai, and K. Wang, "Persistence and extinction of single population in a polluted environment," Electronic Journal of Differential Equations, vol. 108, pp. 1-5, 2004.

[15] J. Wang and K. Wang, "Analysis of a single species with diffusion in a polluted environment," Electronic Journal of Differential Equations, vol. 112, pp. 1-11, 2006.

[16] A. Buonomo, "The periodic solution of van der Pol's equation," SIAM Journal on Applied Mathematics, vol. 59, no. 1, pp. 156-171, 1999.

[17] C. Çelik, "The stability and Hopf bifurcation for a predator-prey system with time delay," Chaos, Solitons and Fractals, vol. 37, no. 1, pp. 87-99, 2008.

[18] Y. Chen, J. Yu, and C. Sun, "Stability and Hopf bifurcation analysis in a three-level food chain system with delay," Chaos, Solitons and Fractals, vol. 31, no. 3, pp. 683-694, 2007.

[19] K. Gopalsamy, Stability and Oscillations in Delay Differential Equations of Population Dynamics, Kluwer Academic, Dordrecht, The Netherlands, 1992.

[20] Y. Kuang, Delay Differential Equations with Applications in Population Dynamics, Academic Press, New York, NY, USA, 1993.

[21] X. Liao, "Hopf and resonant codimension two bifurcation in van der Pol equation with two time delays," Chaos, Solitons \& Fractals, vol. 23, no. 3, pp. 857-871, 2005.

[22] W. M. Liu, "Criterion of Hopf bifurcations without using eigenvalues," Journal of Mathematical Analysis and Applications, vol. 182, no. 1, pp. 250-256, 1994.

[23] A. Maiti, A. K. Pal, and G. P. Samanta, "Effect of time-delay on a food chain model," Applied Mathematics and Computation, vol. 200, no. 1, pp. 189-203, 2008.

[24] A. Maiti, A. K. Pal, and G. P. Samanta, "Usefulness of biocontrol of pests in tea: a mathematical model," Mathematical Modelling of Natural Phenomena, vol. 3, no. 4, pp. 96-113, 2008.

[25] Y. Song, M. Han, and Y. Peng, "Stability and Hopf bifurcations in a competitive Lotka-Volterra system with two delays," Chaos, Solitons \& Fractals, vol. 22, no. 5, pp. 1139-1148, 2004.

[26] Z. H. Wang and H. H. Hu, "Stability of linear time variant dynamic systems with multiple time delays," Acta Mechanica Sinica, vol. 14, no. 3, pp. 274-282, 1998.

[27] R. Xua, Q. Gan, and Z. Ma, "Stability and bifurcation analysis on a ratio-dependent predator-prey model with time delay," Journal of Computational and Applied Mathematics, vol. 230, no. 1, pp. 187-203, 2009.
[28] H. I. Freedman, Deterministic Mathematical Models in Populations Ecology, Marcel Dekker, New York, NY, USA, 1980.

[29] M. Kot, Elements of Mathematical Ecology, Cambridge University Press, Cambridge, UK, 2001.

[30] J. D. Murray, Mathematical Biology, Springer, New York, NY, USA, 1993.

[31] H. Freedman and V. S. H. Rao, "The trade-off between mutual interference and time lags in predator-prey systems," Bulletin of Mathematical Biology, vol. 45, no. 6, pp. 991-1004, 1983.

[32] L. H. Erbe, H. I. Freedman, and V. Sree Hari Rao, "Three-species food-chain models with mutual interference and time delays," Mathematical Biosciences, vol. 80, no. 1, pp. 57-80, 1986.

[33] B. D. Hassard, N. D. Kazarinoff, and Y. H. Wan, Theory and applications of Hopf Bifurcation, Cambridge University Press, Cambridge, UK, 1981. 


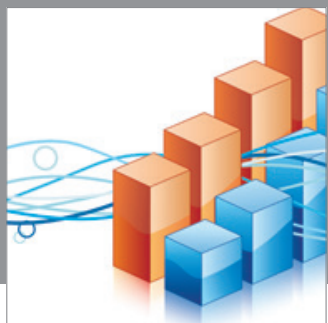

Advances in

Operations Research

mansans

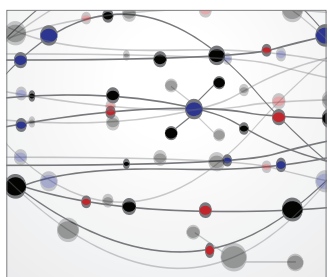

The Scientific World Journal
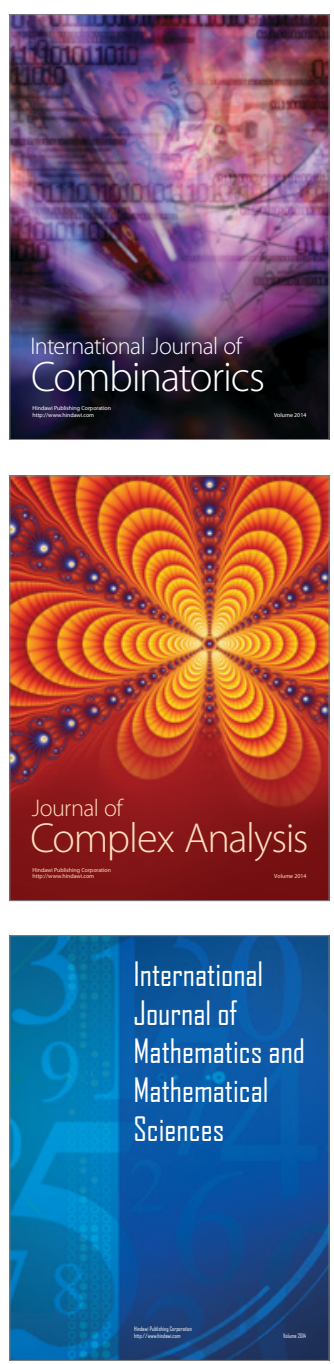
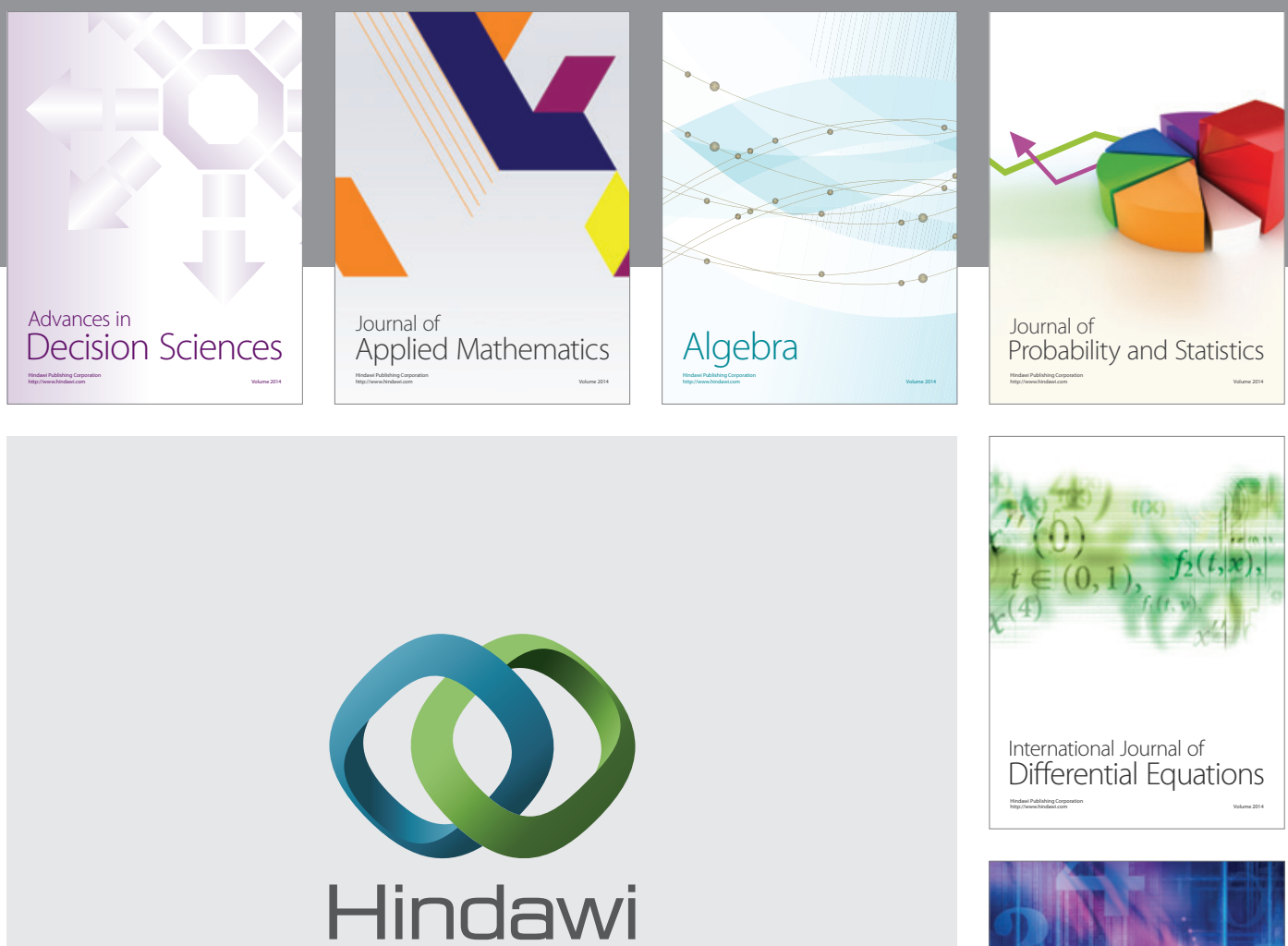

Submit your manuscripts at http://www.hindawi.com
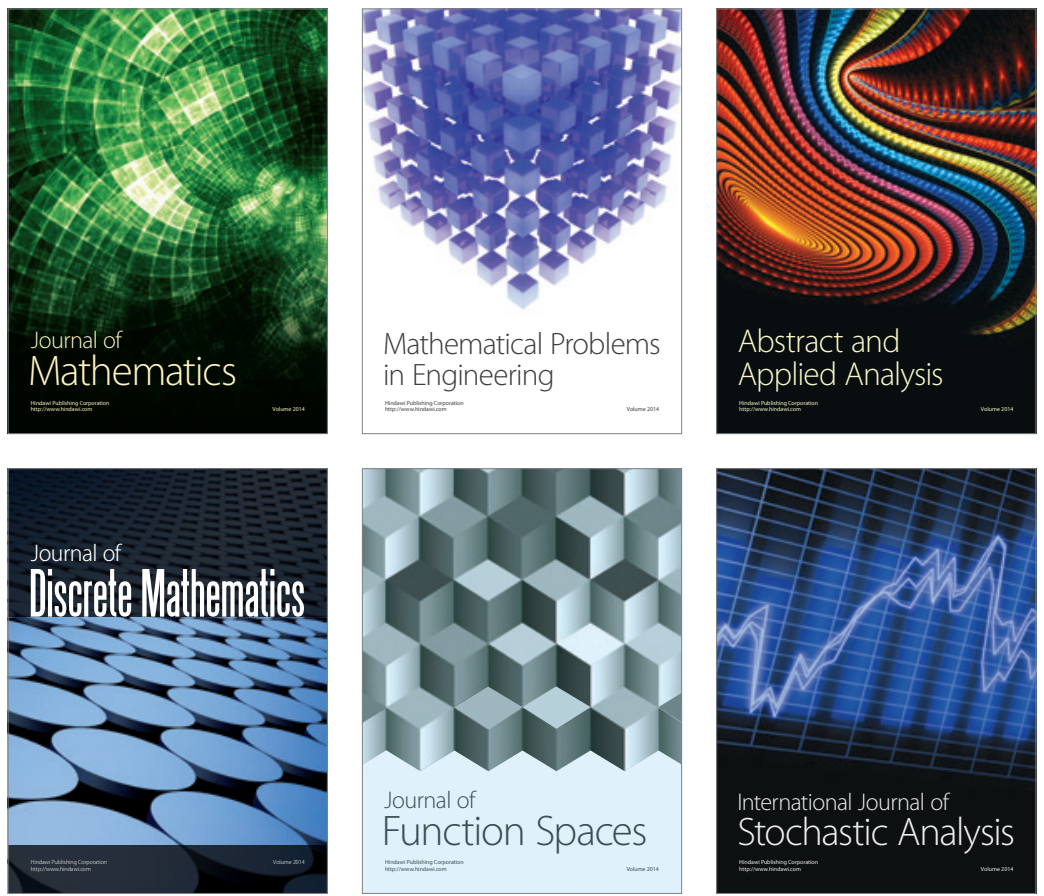

Journal of

Function Spaces

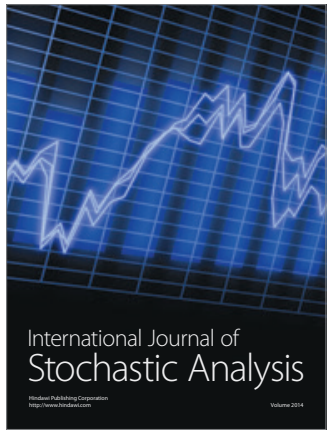

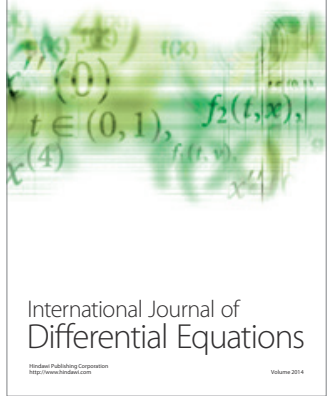
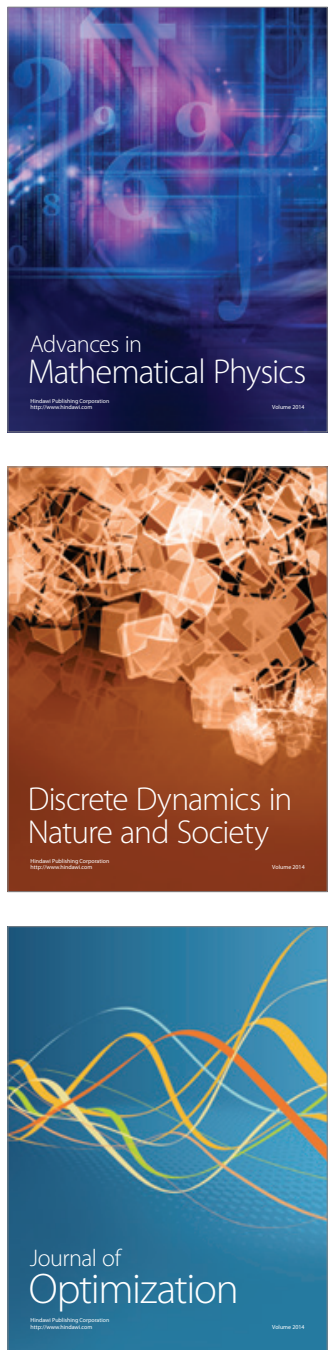